\title{
Two-dimensional van der Waals spinterfaces and magnetic-interfaces
}

DOI:

$10.1063 / 1.5112171$

Document Version

Accepted author manuscript

Link to publication record in Manchester Research Explorer

\section{Citation for published version (APA):}

Dayen, J., Ray, S. J., Karis, O., Vera-marun, I. J., \& Kamalakar, M. V. (2020). Two-dimensional van der Waals spinterfaces and magnetic-interfaces. Applied Physics Reviews, 7(1), 011303. https://doi.org/10.1063/1.5112171

\section{Published in:}

Applied Physics Reviews

\section{Citing this paper}

Please note that where the full-text provided on Manchester Research Explorer is the Author Accepted Manuscript or Proof version this may differ from the final Published version. If citing, it is advised that you check and use the publisher's definitive version.

\section{General rights}

Copyright and moral rights for the publications made accessible in the Research Explorer are retained by the authors and/or other copyright owners and it is a condition of accessing publications that users recognise and abide by the legal requirements associated with these rights.

\section{Takedown policy}

If you believe that this document breaches copyright please refer to the University of Manchester's Takedown Procedures [http://man.ac.uk/04Y6Bo] or contact uml.scholarlycommunications@manchester.ac.uk providing relevant details, so we can investigate your claim.

\section{OPEN ACCESS}




\section{Two-dimensional van der Waals spinterfaces and magnetic- interfaces}

Jean-Francois Dayen ${ }^{1,2}$, Soumya J. Ray ${ }^{3}$, Olof Karis ${ }^{4}$, Ivan J. Vera-Marun ${ }^{5}$ and M. Venkata Kamalakar ${ }^{4} *$

${ }^{1}$ Institut de Physique et Chimie des Matériaux de Strasbourg, Université de Strasbourg, CNRS UMR 7504, 23 rue du Loess, BP 43, F-67 034, Strasbourg Cedex 2, France.

${ }^{2}$ Institut Universitaire de France, 1 rue Descartes, 75231 Paris cedex 05

${ }^{3}$ Department of Physics, Indian Institute of Technology Patna, Bihta 801106, India.

${ }^{4}$ Department of Physics and Astronomy, Uppsala University, Box 516, SE-75120, Uppsala, Sweden.

${ }^{5}$ School of Physics and Astronomy, University of Manchester, Manchester M13 9PL, United Kingdom.

*communication should be sent to

dayen@unistra.fr, ivan.veramarun@manchester.ac.uk, venkata.mutta@physics.uu.se

\section{Abstract}

Two-dimensional (2D) materials have brought fresh prospects for spintronics, as evidenced by the rapid scientific progress made in this frontier over the past decade. In particular, for charge perpendicular to plane vertical magnetic tunnel junctions, the $2 D$ crystals present exclusive features such as atomic-level thickness control, near-perfect crystallography without dangling bonds, novel electronic structure-guided interfaces with tunable hybridization and proximity effects, that lead to an entirely new group of spinterfaces. Such crystals also present new ways of integration of atomically thin barriers in magnetic tunnel junctions and an unprecedented means for developing composite barriers with atomic precision. All these new aspects have sparked interest for theoretical and experimental efforts, revealing intriguing spin-dependent transport and spin inversion effects. Here, we discuss some of the distinctive effects observed in ferromagnetic junctions with prominent $2 D$ crystals such as graphene, hexagonal boron nitride, transition metal dichalcogenides, and how spinterface phenomena at such junctions affect the observed magnetoresistance in devices. Finally, we discuss how the recently emerged 2D ferromagnets bring upon an entirely novel category of van der Waals interfaces for efficient spin transmission and dynamic control through exotic heterostructures.

\section{Table of Contents}

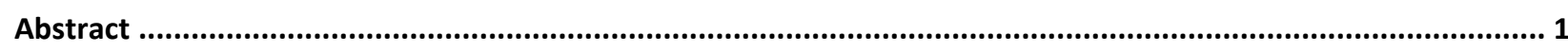

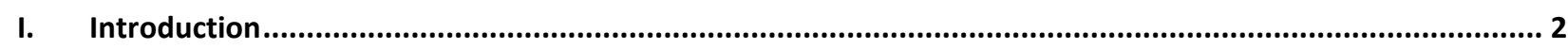

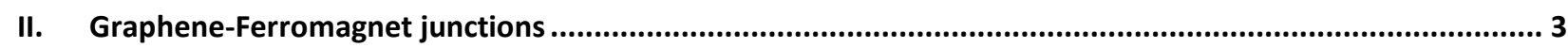
A. Predictions of spin filtering in graphene /FM interfaces............................................................. 4

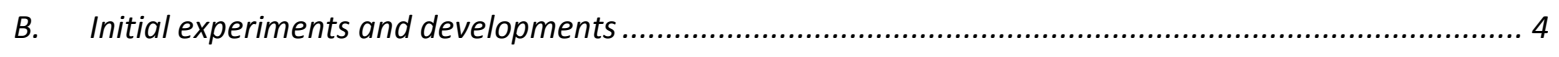

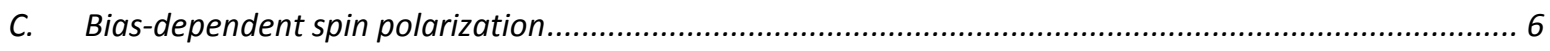

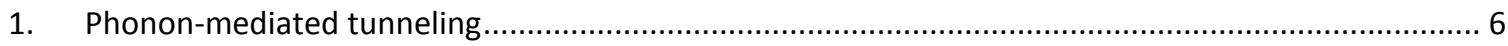

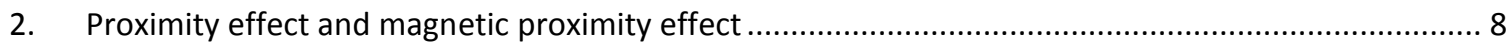

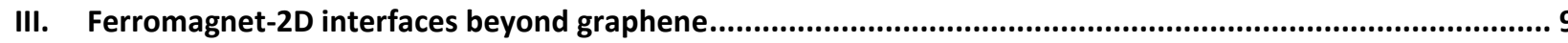

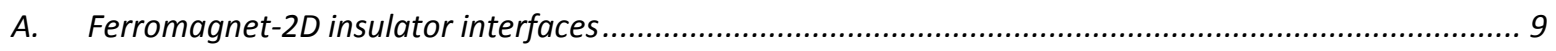

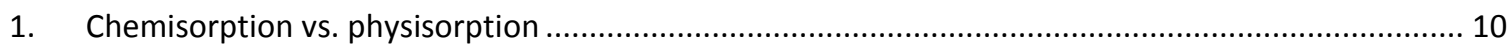

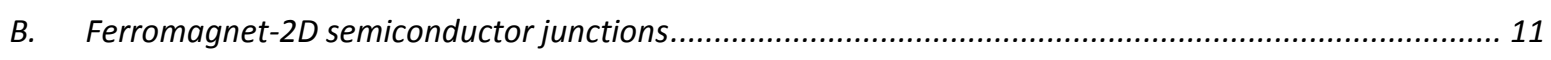

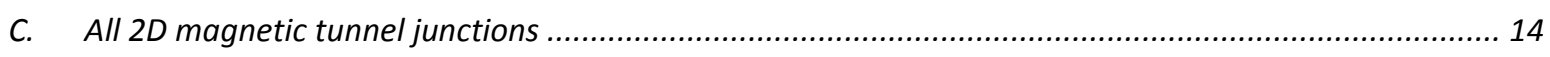

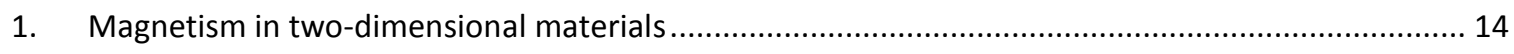

2. Exotic ferromagnetic (eFM) heterostructures for tunable junctions ............................................ 16

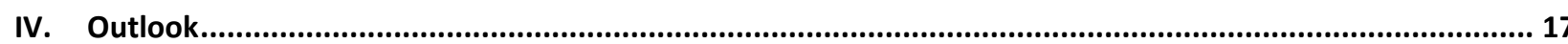

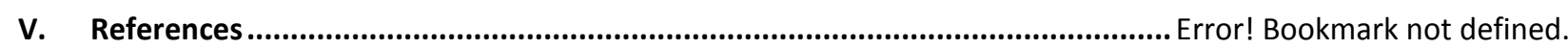




\section{Introduction}

Spintronics presents prospects for spin-based low power memory and logic components, precision sensors and novel power-saving integration architectures. A classic example of a successful spintronic application is the basic structure of the giant magnetoresistive (GMR) and tunnel magnetoresistive (TMR) effects, which has enabled a several thousand-fold enhancements in memory storage capacity, a revolution that served as the base of the present information technology age. A GMR/TMR sensor or a typical spin electronic device consists of a stacked ferromagnet (FM)-Nonmagnet (NM)- ferromagnet (FM) structure, with its efficiency or sensitivity determined by the quality of the FM-NM interfaces. Real interfaces are complex with distinctive hybridization of atomic orbitals of FM and NM, which govern spin polarization and hence are called 'spinterfaces.' In a broader sense, a spinterface ${ }^{1}$ is a spin-resolved interface created at a ferromagnet and a low dimensional material junction due to hybridization or contiguity effects. While the term 'spinterface' was coined in 2010 to describe spin polarization due to interface between ferromagnetic layers and organic molecules, it was already evident in 1999 that the ferromagnet/barrier interface guides spin polarization and the TMR response in magnetic tunnel junctions (MTJs) ${ }^{2}$. The spin-polarized transport in such interfaces is distinct to the individual materials forming the junction. The efficiency to inject or detect spin-polarized electrons across such an interface is affected by several features such as interface roughness, defects, impurities, and mismatch in conductivity of the materials forming the spinterface. Research in traditional magnetic tunnel junctions and physics of interfaces ${ }^{3}$ has led us to present-day highly efficient data storage and magneto-sensing technologies, enabling the information technology age. Many exciting observations have been reported in spin-dependent tunneling ${ }^{3}$, including bias-controlled inversion of $\mathrm{TMR}^{4}$. In conventional magnetic tunnel junctions prepared with oxides as a spacer, achieving high crystallinity and smooth interfaces is a great challenge. Such a challenge can be fundamentally mitigated with a new class of graphene and graphene-like two-dimensional (2D) crystals, which provide fresh impetus for studying novel spinterfaces created by such high quality atomically thin crystals with FM. In this review, we expand the spinterface concept introduced for ferromagnet-molecular junctions, to new spinterface phenomena occurring at FM-2D material junction (Fig. 1).

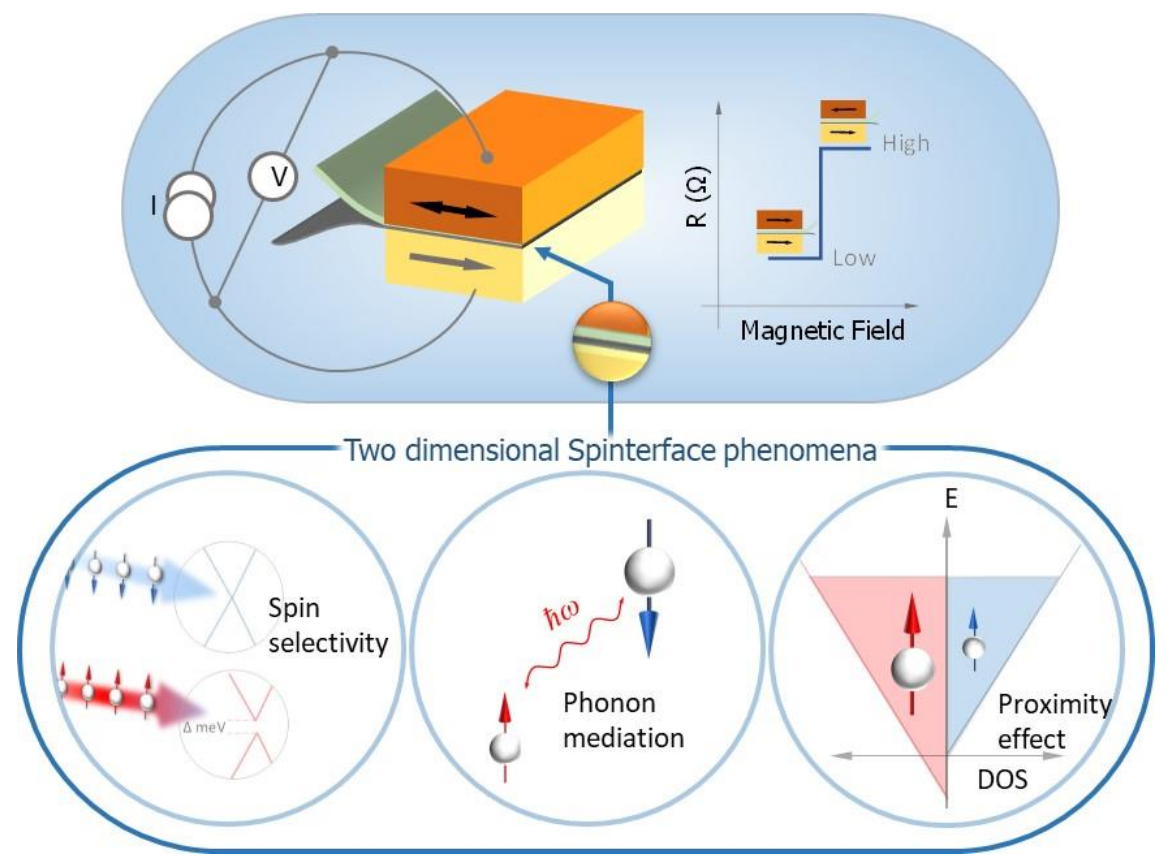

Figure 1. A basic spin valve structure consisting of the $2 \mathrm{D}$ layers as spacers. The Resistance $\mathrm{R}$ vs Magnetic field shows switching between the high and low voltage levels measured for parallel and anti-parallel configurations of the magnets. The lower panel shows three important spin transmission phenomena occurring at the 2D material|Ferromagnet interfaces.

In contrast to the top-down approach of obtaining smooth thin films of 3D materials, the 2D crystals provide close to ideal means for creating atomically thin layers in devices. These crystals occur in layers and do not exhibit dangling bonds, or major defects, and offer new ways of epitaxy with ferromagnetic layers. The wide variety of band structure features such as zero bandgap in graphene, high bandgap 2D insulators, tunable 
direct to indirect bandgaps in semiconducting crystals with intrinsic spin-orbit coupling and tunable electronic behavior, provide new knobs for controlling the magnetoresistance. In addition, proximity with ferromagnets induces changes in the band structure of such atomically thin spacers and can result in spin-selective transmission across such interfaces. The 2D crystals can also be grown in large-scale, directly on ferromagnetic metals, and play an important role as an oxide-passivating layer. Spacers comprising of such layered materials thus present enticing means to unlock new possibilities with magnetic junctions. The hybridization at the interfaces with magnets or the Van der Waals nature of the contacts allows one to explore new physics of proximity effects, inaccessible with conventional materials. Here, we highlight some unique spin filtering, bias dependent observations accompanied by 2D materials spacers that started with graphene and progressed with other important crystals such as hexagonal boron nitride (hBN) and transition metal dichalcogenides in vertical spin valves. Followed by this, we discuss the recent discoveries of $2 \mathrm{D}$ ferromagnets and how all-2D crystalbased magnetic tunnel junctions could lead to new benchmark for performance and new functional spin active heterostructures.

\section{Graphene-Ferromagnet junctions}

Graphene was the first 2D crystal where vertical spin transport was reported and extensively investigated. In general, graphene is an outstanding medium for lateral spin transport, primarily due to its high carrier mobility and low-spin orbit coupling, which led to the emergence of graphene spintronics. ${ }^{5-9}$ In addition to lateral spin transport, spin transmission through layers of graphene in contact with ferromagnets has shown distinctive prospects, with initial theoretical works ${ }^{10-12}$ predicting large spin-filtering efficiencies in FM| $n$ Gr interfaces. Over the past decade, experiments involving graphene as a spacer in the current perpendicular to the plane (CPP) spin valves have shown several important results such as spin filtering, enhancements in magnetoresistance and bias dependent selective spin transmission. In this part, we will discuss developments of various graphene-based vertical spin valves, focusing on interface phenomena and the conditions behind the observed figures of merits, including their bias dependencies.

a

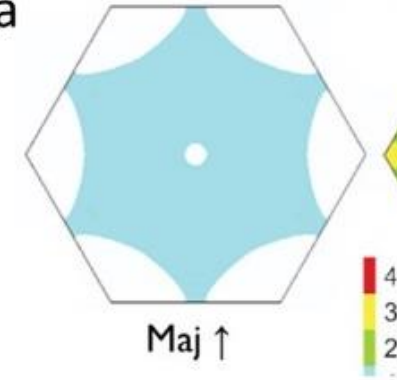

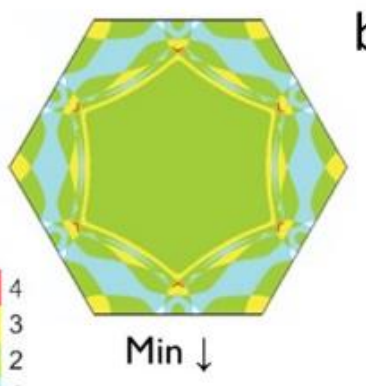

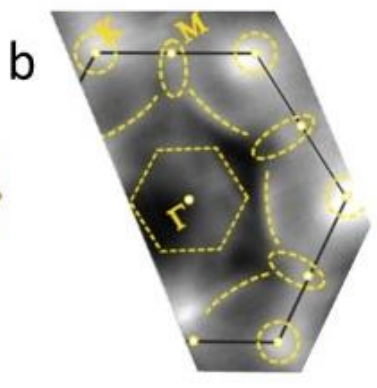

C

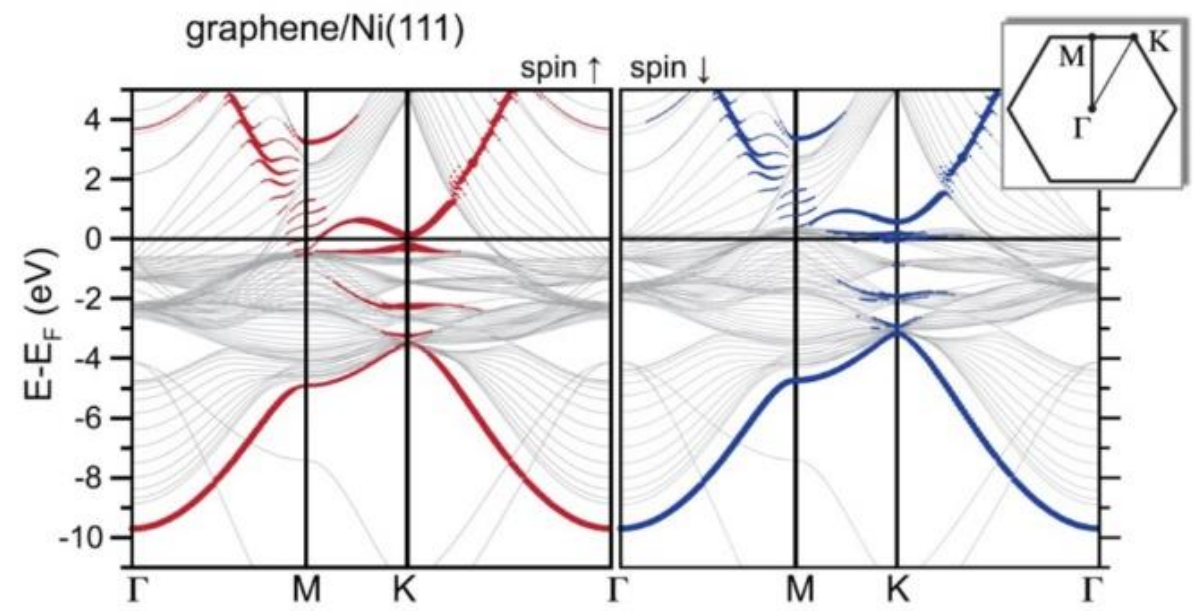

Figure 2. (a) Fermi surface projections onto the (111) plane of majority and minority spins for fcc Nikel ${ }^{10}$. Only minority spin carriers are expected to contribute at the $\Gamma$ point. Reprinted with permission from Karpan, V. M. et al., Phys. Rev. Lett. 99, 176602 (2007). Copyright 2007 American Physical Society. (b) Constant energy cut at the Fermi energy of ARPES analysis of $\mathrm{Gr} / \mathrm{Ni}(111)$ interface. Increased photoemission intensity is observed at the $\mathrm{K}$ points, around the $\mathrm{M}$ points, and a diamond shape regions close to the $\Gamma$ point ${ }^{15}$. (c) Spin-resolved electronic band structure of graphene on Ni(111) (inset shows the Gr Brillouin-zone with corresponding $\Gamma-\mathrm{M}$ - K- $\Gamma$ direction) showing more complex spin polarized structure including at the $\mathrm{M}$ point at the Fermi energy ${ }^{15}$. Adapted with permission from Dedkov, Y. S. and M. Fonin, New J. Phys. 12, 125004 (2010). 


\section{A. Predictions of spin filtering in graphene|FM interfaces}

In seminal works ${ }^{10,11}$, through first-principle calculations, Karpan et al. predicted that large spin filtering efficiency should occur at the interface between few-layer graphene and ferromagnetic electrodes of (111) fcc or (0001) hcp nickel or cobalt. The underlying reason behind this efficiency is that, close to the Dirac point, the electronic structures of a ferromagnet (FM) and graphene (Gr) only overlap for the minority spin direction in the parts of the reciprocal space corresponding to the $\mathrm{K}$ and $\mathrm{K}^{\prime}$ points of graphene. Therefore, minority spinpolarized electrons preferably transmit from the FM surface into graphene. Magnetoresistance study allows for probing the amplitude of the spin signals. Tunnel magnetoresistance ('TMR') is usually used to quantify MTJ efficiency. In the following, we will use the so-called pessimistic definition of $T M R: T M R=\left(R_{A P}-R_{P}\right) / R_{A P}$, with $R_{P}$ and $R_{A P}$ representing the junction resistance with parallel (P) and anti-parallel (AP) alignment of electrodes magnetizations respectively. TMR signals up to $100 \%$ have been predicted for ideal Gr/FM junction. As we will see later in this review, this prediction forms the fundamental basis in understanding the role played by the $\mathrm{Gr} / \mathrm{FM}$ interface. However, as we will elaborate in this review, the Gr/FM interface can be rather complex, and different spin-resolved properties can vary depending on the interaction between graphene and ferromagnet, which could range from a robust covalent coupling, Van der Waals interaction to proximity induced interaction.

\section{B. Initial experiments and developments}

Experimental evidence of the spin-resolved properties of the Gr/FM interface came as early as 2008, with the physicochemical characterization of the CVD Gr/Ni(111) interface and the study of its magnetic properties ${ }^{13}$. Complementary studies allowed to characterize the electronic structure of $\mathrm{Ni}(111) / \mathrm{Gr}$ interface, its physicochemical composition, and its spintronics properties (for detailed analysis on $\mathrm{Ni} / \mathrm{Gr}$ interface see references ${ }^{14,15}$ ). These pioneering reports essentially revealed that $\mathrm{Gr}$ passivates the Nickel surface from oxidation, and new spintronics properties are promoted at the $\mathrm{Gr} / \mathrm{Ni}$ interface. Mostly, the $\pi$-orbitals of the graphene are magnetically polarized due to hybridization with the $\mathrm{Ni}$-3d states of the substrate, leading to spinpolarized interface states, mainly at the $\Gamma, K$ and $M$ points (close to the Fermi energy, see Figure 2.b-c).

The initial transport measurements by Mohiuddin et al. ${ }^{16}$ (also in 2008), showed MR 0.4\% in exfoliated graphene layers sandwiched between two Py electrodes. Using an additional $2 \mathrm{~nm}$ Au layer to prevent the bottom Py electrode from potential oxidation during the graphene transfer, the Py|Au|Gr|Py structure revealed an enhanced $M R \sim 5 \%$, which confirmed the significance of $F M / G r$ interface quality. Apart from similar studies on exfoliated graphene (see Table 1), in 2012, Cobas et al. demonstrated the large scale processing of $\mathrm{NiFe} / \mathrm{Gr} / \mathrm{NiFe}$ MTJ with the implementation of large-area CVD graphene grown over Cu substrate by a PMMA assisted wet-transferred over the Nife bottom electrodes ${ }^{17}$. Slightly non-linear I-V characteristics were reported with positive MR signals up to $2 \%$ and MR amplitude decreasing monotonically and non-linearly with the bias voltage (up to $120 \mathrm{mV}$ ), which was interpreted as spin relaxation via phonons and magnons interactions. Low MR was attributed to roughness and poly-crystallinity of the bottom FM layer and probable oxidation of the unprotected NiFe layer during the wet transfer step. The same year, Dlubak et al. ${ }^{18}$ reported the first application of CVD graphene grown on Nickel substrate into MTJ, circumventing the need for any transfer step, known to be detrimental to the $\mathrm{FM} / \mathrm{Gr}$ interface quality. In these $\mathrm{MTJs}$, an $\mathrm{Al}_{2} \mathrm{O}_{3}$ tunnel barrier was employed in $\mathrm{FM}|\mathrm{CVD} \mathrm{Gr}| \mathrm{Al}_{2} \mathrm{O}_{3} \mid \mathrm{FM}$ junction and $\mathrm{MR}$ amplitude as large as $-10.8 \%$ was reported. These high MR signals, together with XPS analysis, demonstrated that graphene protected ferromagnetic electrodes (GPFE) can be used as oxygen passivated electrodes for spintronics devices. The negative sign of MR signal and the asymmetric configuration of MTJ electrodes allowed for attributing a negative spin polarization to the spin interface $\mathrm{Gr} / \mathrm{Ni}$ (described later). The promising spin filtering properties of GPFE have been recently protracted to Co|CVD Gr interfaces, with an observation of Gr-assisted passivation of the Co electrode, spin-polarized bandstructure mapping of the $\mathrm{Gr} / \mathrm{Co}$ interface by ARPES measurements ${ }^{19}$, and recent demonstration of negative MR and spin polarization through magnetotransport study of $\mathrm{Gr} / \mathrm{Co}$-based MTJ ${ }^{20}$.

These initial studies showed the possibility of graphene-based magnetic tunnel junctions while suggesting the need for better fabrication strategies to avoid oxidation of the FM electrode contacting the graphene barrier, improve the graphene/electrode interface (roughness, homogeneity), and generate better insights into the $\mathrm{Gr} / \mathrm{FM}$ interface role in the device properties. Several subsequent reports circumventing the exposure of FM to air or solvents demonstrated improved $\mathrm{MR}^{21-23}$. For details of such technical issues and processes, we refer the readers to dedicated discussions ${ }^{24,25}$. In Table-I, we summarize a list of studies in the area performed on various spin-valve junction structures prepared using different fabrication methods. A majority of these reports involving transferred monolayer graphene showed $M R \sim 0.1-5 \%$, with distinct improvements in hybrid 
architectures demonstrating $\mathrm{MR}>10 \%{ }^{18,26,27}$, which are still much smaller than theoretical expectations ${ }^{10,11}$. In particular, Cobas et al., have reported spin filtering at $\mathrm{FM} / \mathrm{Gr}$ interfaces demonstrating $-12 \%$ of MR with $\mathrm{NiFe}(111)|\mathrm{Gr}| \mathrm{Fe}$ MTJ. Considering a two channels model, the authors extrapolated a NiFe(111)/Gr interface spin polarization $\sim 80 \%$ at low temperature. Overall, the continuous efforts and progress demonstrated that spin filtering in $\mathrm{Gr}$ based ferromagnetic junctions show great potential for enhancing figures of merit. Most studies performed with exfoliated Gr report positive spin polarization, while negative spin polarization is observed in several CVD Gr based MTJ, as predicted by Karpan ${ }^{11}$. As we will see, interestingly, bias voltage inversion of TMR polarity has been observed both for CVD Gr/Ni interface ${ }^{28}$ and for annealed exfoliated Gr /FM interfaces $^{23}$. Such behavior points out the critical role of the nature of the Gr/FM interface. In the case of exfoliated or transferred graphene over FM substrate, the first graphene layer interacts mostly via Van der Waals interactions (physisorption). In case of an epitaxial device (CVD graphene grown over FM substrate without post-transfer), the $\mathrm{Gr}$ is in covalent interaction (chemisorption) with the $\mathrm{FM}$ interface and the $\mathrm{FM} / \mathrm{Gr}$ distance is much shorter ( $2 \AA$ ) than for exfoliated or transferred Gr/FM interface ( 3-4 $\AA$ ). Hybridization is then expected to occur for Gr grown over FM substrate ${ }^{11}$ and should lead to new spin filtering properties.

Table-1: Experiments involving $\mathrm{Gr}$ as a spacer in MTJs. In calculating $\mathrm{P}$ in ref. ${ }^{18,26,29}$, the polarization of $\mathrm{Co} / \mathrm{Al}_{2} \mathrm{O}_{3}$ interface was taken as as $+32 \%$.

\begin{tabular}{|c|c|c|c|c|c|}
\hline MTJ structure & Ref. & $\begin{array}{l}\text { Layer } \\
\text { thickness }\end{array}$ & $\begin{array}{c}\text { MR (\%) } \\
\text { (Temperature) }\end{array}$ & $\begin{array}{l}\text { Polarization } \\
\text { (P) }\end{array}$ & $\begin{array}{c}\text { 2D layer Growth } \\
\text { Method }\end{array}$ \\
\hline $\mathrm{NiFe}|\mathrm{Gr}| \mathrm{NiFe}$ & 16 & Monolayer & $0.4 \%(300 \mathrm{~K})$ & $4.5 \%$ & Exfoliated Gr \\
\hline $\mathrm{NiFe}|\mathrm{Au}| \mathrm{Gr} \mid \mathrm{NiFe}$ & & & $4 \%(300 K)$ & $14 \%$ & \\
\hline $\mathrm{Ni}\left|\mathrm{Al}_{2} \mathrm{O}_{3}\right| \mathrm{Gr} \mid \mathrm{Co}$ & 30 & Monolayer & $-0.4 \%(300 K)$ & - & $\begin{array}{l}\text { Exfoliated Gr, wet } \\
\text { transfer. }\end{array}$ \\
\hline Co|Gr|NiFe & 23 & $1,2,3,4$ layers & $\begin{array}{l}-0.7 \text { to }+1 \%(10 \mathrm{~K}) \\
\text { Bias-induced inver } \\
\text { Magnetic proximit }\end{array}$ & $\begin{array}{l}7 \% \\
\text { in pol. } \\
\text { fect. }\end{array}$ & $\begin{array}{l}\text { Exfoliated Gr. } \\
\mathrm{MTJ} \text { annealed at } \\
300^{\circ} \mathrm{C} \text { in } \mathrm{Ar} / \mathrm{H} \\
\text { atmosphere. }\end{array}$ \\
\hline $\mathrm{NiFe}|\mathrm{Gr}| \mathrm{Co}$ & 17 & Monolayer & $\begin{array}{l}2 \%(4 \mathrm{~K}) \\
0.4 \%(300 \mathrm{~K})\end{array}$ & $\begin{array}{l}10 \% \\
4.5 \%\end{array}$ & \multirow{6}{*}{$\begin{array}{l}\text { CVD Gr over Cu, } \\
\text { Wet transferred }\end{array}$} \\
\hline ColGr|Co & 31 & 1,2 layers & $\begin{array}{l}1 \%(1.5 \mathrm{~K}) \\
0.7 \%(300 \mathrm{~K})\end{array}$ & $\begin{array}{l}7 \% \\
5.9 \%\end{array}$ & \\
\hline $\mathrm{NiFe}|\mathrm{Gr}| \mathrm{NiFe}$ & 32 & 1,2 layers & $\begin{array}{l}0.5 \%(10 \mathrm{~K}) \\
0.27 \%(300 \mathrm{~K})\end{array}$ & $\begin{array}{l}5 \% \\
3.7 \%\end{array}$ & \\
\hline ColGr|Co & 33 & 1,2 layers & $\begin{array}{l}1 \%(3 \mathrm{~K}) \\
0.9 \%(300 \mathrm{~K})\end{array}$ & $\begin{array}{l}7 \% \\
6.7 \%\end{array}$ & \\
\hline $\mathrm{NiFe}|\mathrm{Gr}| \mathrm{Co}$ & 21 & Monolayer & $\begin{array}{l}3.4 \%(6.1 \mathrm{~K}) \\
1.7 \%(300 \mathrm{~K})\end{array}$ & $\begin{array}{l}13 \% \\
9.2 \%\end{array}$ & \\
\hline $\begin{array}{l}\mathrm{NiFe}\left|\mathrm{Al}_{2} \mathrm{O}_{3}\right| \mathrm{Gr} \mid \mathrm{Co} \\
\mathrm{NiFe}|\mathrm{Gr}| \mathrm{Co}\end{array}$ & 34 & Monolayer & $\begin{array}{l}-1.6 \%(10 \mathrm{~K}) \\
0.23 \%(300 \mathrm{~K}) \\
\text { Negative MR with }\end{array}$ & $\begin{array}{l}- \\
\mathrm{Al} \\
\mathrm{I}_{2} \mathrm{O}_{3} / \mathrm{Gr}\end{array}$ & \\
\hline $\mathrm{NiFe}|\mathrm{Gr}| \mathrm{NiFe}$ & 22 & $1,2,3,4$ layers & $4.6 \%(4.2 \mathrm{~K})$ & $15 \%$ & $\begin{array}{l}\text { Exfoliated, Wet } \\
\text { transferred }\end{array}$ \\
\hline $\mathrm{Ni}|\mathrm{Gr}| \mathrm{Co}$ & 35 & Monolayer & $0.16 \%(300 \mathrm{~K})$ & $2.8 \%$ & \\
\hline $\mathrm{Ni}|\mathrm{Au}| \mathrm{Gr} \mid \mathrm{Co}$ & & & $0.57 \%(300 \mathrm{~K})$ & $5.3 \%$ & \\
\hline $\mathrm{Ni}\left|\mathrm{Al}_{2} \mathrm{O}_{3}\right| \mathrm{Gr} \mid \mathrm{Co}$ & & & $\begin{array}{l}-0.52 \%(300 \mathrm{~K}) \\
\text { Negative pol. with } \\
\text { tunneling regime. }\end{array}$ & $\mathrm{Al}_{2} \mathrm{O}_{3} \mid$ Gr interface in & $\begin{array}{l}\text { CVD Gr over Cu, } \\
\text { Wet transferred }\end{array}$ \\
\hline $\mathrm{Ni}|\mathrm{Gr}| \mathrm{Al}_{2} \mathrm{O}_{3} \mid \mathrm{Co}$ & 18 & 2 to 5 layers & $-10.8 \%(1.4 \mathrm{~K})$ & $-16 \%$ & CVD Gr over Ni, \\
\hline
\end{tabular}


Phonon-assisted tunneling.

$\mathrm{P}<0$ channel at $\mathrm{K}$ point.

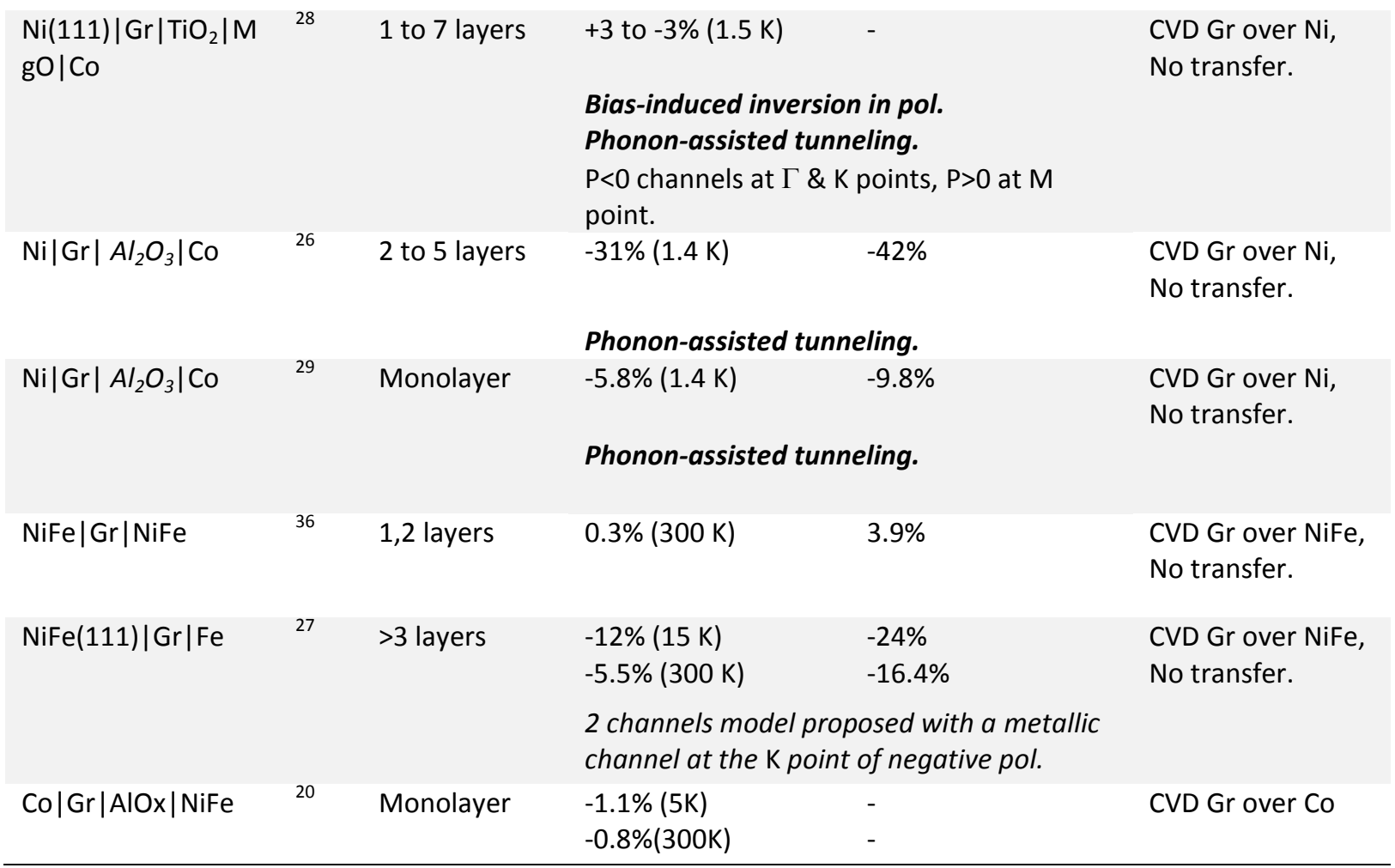

No transfer.

VD Gr over $\mathrm{Ni}$

CVD Gr over Ni, No transfer.

CVD Gr over $\mathrm{Ni}$, No transfer.

CVD Gr over NiFe, CVD Gr over NiFe,

.

\section{Bias-dependent spin polarization}

Apart from a high magnetoresistance that graphene-based magnetic junctions can display, tunability of the magnitude, as well as the sign of magnetoresistance (interface polarization), are exciting aspects. In the following, we briefly discuss two specific mechanisms that result in characteristic bias dependences of TMR.

\section{Phonon-mediated tunneling}

In the Jullière's model ${ }^{37}$, the TMR amplitude is proportional to the product of each interface polarity $\left(P_{1}, P_{2}\right.$, which are considered identical if both electrodes are made of the same material) as $T M R=2 P_{1} P_{2} /\left(1-P_{1} P_{2}\right)$. Therefore, spin valves of FM/Gr/FM type designed with symmetric interfaces made of identical FM material, prevent for addressing the polarity of the FM/Gr interface spin polarization. This also underlines the need for a hybrid spacer device architecture allowing to better explore the existence of spin filtering effects. As shown in Table-I, such FM/Gr hybrid/oxide barrier junction not only displays enhanced MR but also provides an opportunity to investigate the bias dependence of $\mathrm{FM} / \mathrm{Gr}$ interfaces. The $\mathrm{FM} / \mathrm{Gr}$ interface is central for deciding the magnitude and sign of spin polarization, and developing asymmetric interface MTJ structures helps one to determine interface phenomena. The lattice mismatch of graphene/Ni(111) and graphene/Co(0001) interfaces is close to $1 \%$, one of the smallest among the MTJs studied so far. As discussed by Karpan and coworkers ${ }^{10,11}$, in case of highest quality Gr/FM interfaces, a strong hybridization of carbon $\pi$ orbitals with $\mathrm{Ni}$ (or $\mathrm{Co}$ ) surface atoms is expected. Few-layer graphene could, on one hand, act as a tunnel barrier for majority spin electrons providing from FM electrodes, and on the other hand, promote selected transmission of minority spin carriers at low energy at $\mathrm{K}$ point. At higher energy (bias voltage), a larger area of the Gr/FM Fermi surface could be explored and should reveal a more complex spin-polarized band structure triggered by Gr/FM interactions. Dlubak et al. reported the first magnetotransport study on MTJ implementing a $\mathrm{Ni} /$ few-layer CVD Gr electrode into an asymmetric Ni/CVD-Gr/AI $\mathrm{O}_{3} / \mathrm{Co}$ stack. The IV-characteristic of such junctions showed clear non-ohmic behavior (Figure 3a), confirming that electrons are tunneling through the MTJ, characterized by a textured dip in the junction differential conductance $G(V)=d I(V) / d V$ within the voltage range $\pm 60 \mathrm{mV}$. This conductance dip was ascribed to the opening of phonon-assisted inelastic tunneling channel, involving $\Gamma \rightarrow K$ graphene out-of-plane acoustic phonon mode at resonant energy of 60 meV (see Figure $3 d, e)^{38}$. MR signatures down to $-10.8 \%$ were measured for $100 \mathrm{mV}$ bias amplitude MR (Figure $3 b$ ). The 
negative sign of the MR was assigned to the spin polarity of the $\mathrm{Ni} / \mathrm{Gr}$ interface at the $\mathrm{K}$ point, probed by phonon-assisted tunneling processes ${ }^{39}$ under $100 \mathrm{mV}$ bias voltage. This was the first transport experiment results addressing the negative spin polarity at the $\mathrm{K}$ point of the $\mathrm{Gr} / \mathrm{Ni}$ interface. The same group improved recently the device performance while implementing optimized atomic layer deposition (ALD) growth of $\mathrm{Al}_{2} \mathrm{O}_{3}$ tunnel barrier with a sub-nm thickness (see Table I, reference ${ }^{26}$ and ${ }^{29}$ ).

a

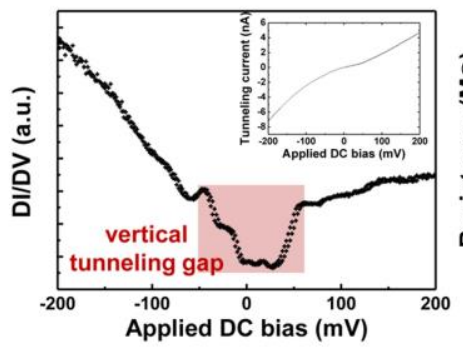

b

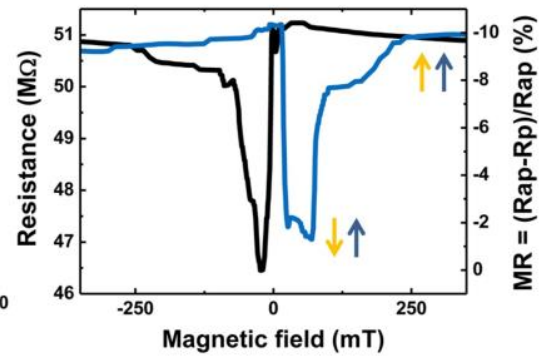

C

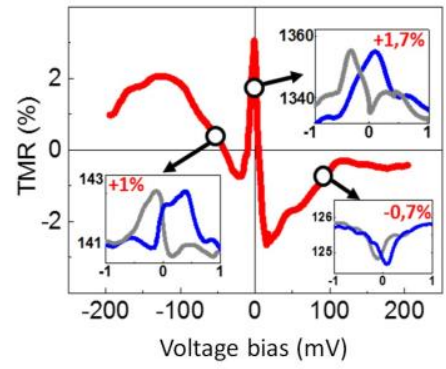

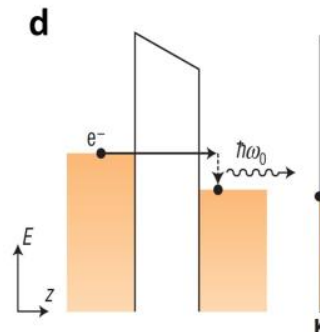
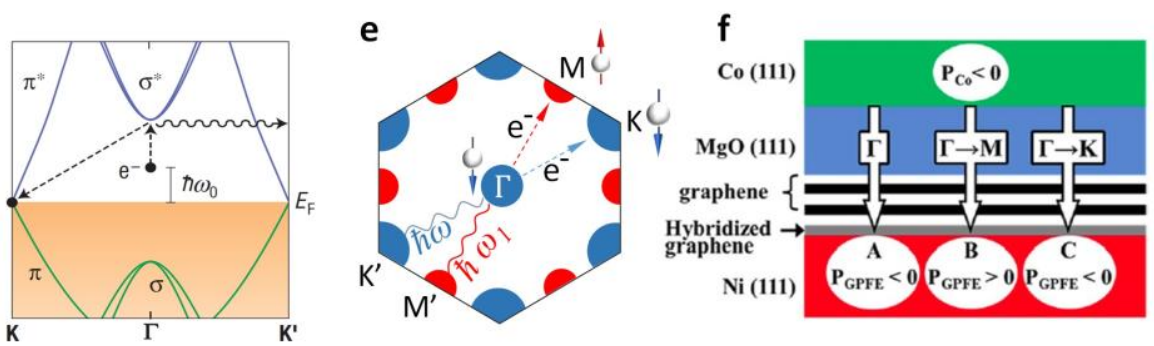

Figure 3. (a) Differential conductance versus voltage measurement of $\mathrm{Co} / \mathrm{Al}_{2} \mathrm{O}_{3} / \mathrm{CVD} \mathrm{Gr} / \mathrm{Ni} \mathrm{MTJ}$ (inset: corresponding I-V curve). A textured gap is observed of full width $\sim 120 \mathrm{meV}$, characterized as phonon-assisted tunneling fingerprints. ${ }^{18}$ (b) Magnetoresistance of the same $\mathrm{Co} / \mathrm{Al}_{2} \mathrm{O}_{3} / \mathrm{CVD} \mathrm{Gr} / \mathrm{Ni} \mathrm{MTJ}$ showing negative MR signal under $-100 \mathrm{mV}$ applied bias. ${ }^{18}$ Adapted with permission from Dlubak, B. et al., ACS Nano 6, 10930 (2012). (c) Bias dependence of the MR signal of $\mathrm{Co} / \mathrm{MgO} / \mathrm{CVD} \mathrm{Gr} / \mathrm{Ni}(111)$. The insets show magnetoresistance loops (resistance in $\mathrm{k} \Omega$, magnetic field in Tesla) measured at different bias voltages, illustrating the sign reversal of TMR. ${ }^{28}$ Reproduced from Godel, F. et al., Appl. Phys. Lett. 105, 152407 (2014) with the permission of AIP Publishing. (d) Band diagram schematics of inelastic electron tunneling due to excitation with energy $\hbar \omega_{0}{ }^{38}$ and band structure schematics of wave-vector-dependent inelastic tunneling mechanism involving graphene phonon modes near the $\mathrm{K}$ point in reciprocal space. ${ }^{38} \mathrm{~A}$ virtual electronic state is experienced at $\mathrm{K}$ point into the graphene $\sigma^{*}$ band, before relaxing into an available $K$ point state on the $\pi$ band through the emission of $\Gamma \rightarrow K^{\prime}$ outof-plane phonon (to satisfy momentum conservation). Adapted with permission from Zhang, Y. et al., Nat. Phys. 4, 627 (2008). Copyright 2008 Nature Publishing Group. (e), (f) Spin-polarized band structure of Gr over Ni(111) deduced from $\operatorname{TMR}(\mathrm{V})$ measurements of ref. ${ }^{28}$ with the majority (respective minority) spin density of state in red (rep. in blue). The transport mechanism (shown in $\mathrm{f}$ ) is illustrated by arrows (straight arrows indicate the inelastic tunneling process, with the activated phonon illustrated by waved arrows).

Taking a similar approach, Godel et al. ${ }^{28}$ addressed carefully the bias dependence of the MR properties of $\mathrm{Ni}(111) /$ few layers CVD Gr electrode implemented into an asymmetric (111)Ni/CVD-Gr/MgO/Co MTJ. The voltage dependence of MTJ derivative conductance showed systematically non-ohmic behavior, with RA junction product of 10-100 M $\Omega . \mu \mathrm{m}^{2}$ confirming the good quality of the tunnel barrier, further, characterized by a dip reminiscent of the hybridized $\mathrm{Gr} / \mathrm{Ni}$ interface band structure as predicted by ab-initio calculation ${ }^{11}$. The overall bias dependency of the MR signal MR(V) was qualitatively identical for all devices, with several sign reversals that can be decomposed into three distinct bias voltage regimes (Figure $3 \mathrm{c}$ ). Based on ab initio calculations of the $\mathrm{Ni} / \mathrm{Gr}$ interface spin-polarized band structure ${ }^{11}$ and its phonon dispersion ${ }^{40}$, the authors identified three spin-polarized channels related to the $\Gamma, \mathrm{M}$, and $\mathrm{K}$ points of the hybridized Gr Brillouin zone ${ }^{28}$, in good agreement with ARPES analysis of $\mathrm{Gr} / \mathrm{Ni}$ interface ${ }^{13}$ (Figure 2.b). The $\Gamma$ point channel occurs by direct tunneling, while phonon-assisted tunneling allows for conduction through the $M$ point (at resonant energy of $40 \mathrm{meV}$ ) and the $\mathrm{K}$ point (at resonant energy of $60 \mathrm{meV}$ ). The asymmetric configuration of the device FM electrodes allowed for identifying the spin polarization of each channel. The tunneling spin polarization of the GPFE changes from negative in the $\Gamma$ and $K$ channels to positive in the $M$ channel, the latter dominating conduction at intermediate voltage values (Figure $3 \mathrm{f}$ ), which is in good agreement with theoretical prediction ${ }^{41}$ (see Figure 1c). Interestingly, phonon mediated inelastic tunneling at $\mathrm{M}$ point was recently observed also on $\mathrm{Gr}$ over $\operatorname{Ir}(111)$ substrate $^{42}$. These results experimentally revealed the complexity of the spin polarization of 
hybridized graphene and demonstrated spin injectors which can be switched from negative to positive spin polarization as a function with a bias voltage.

\section{Proximity effect and magnetic proximity effect}

Proximity effects offer tantalizing opportunities for engineering the electronic structure of 2D materials. Here, the capability of achieving atomically thin materials, together with improved interface quality, enables us to transform a 2D material by acquiring the properties of its neighbors. The 2D material can become superconducting, magnetic, topologically non-trivial, or enhance its spin-orbit coupling, realizing properties not present in any of the individual components of the structure ${ }^{43}$. A prototypical example of such a proximity effect is exhibited in a van der Waals structure from graphene on top of a transition-metal dichalcogenide, which is relevant to spintronics ${ }^{44}$. In these structures the Dirac cone lies within the gap of the transition-metal dichalcogenide, preserving the identity of the $\pi$-bands while acquiring a remarkably large spin-orbit coupling. The latter has been shown experimentally to reach up to the order of $10 \mathrm{meV}$ and to lead to the appearance of the spin Hall effect in graphene ${ }^{45,46}$. Furthermore, first-principle calculations have also proposed these structures could be used to enable direct optical spin injection in graphene ${ }^{47}$, which was recently demonstrated experimentally ${ }^{48,49}$. Besides spin-orbit coupling, another property accessible to graphene via proximity effect, and relevant to spintronics, is magnetism. Although one can introduce Zeeman coupling by applying a large external magnetic field, proximity exchange fields can introduce much stronger effects ${ }^{50-52}$, potentially without significantly altering the band structure. Importantly, the short-range nature of proximity effects results in the creation of a large exchange field mostly within the outermost 2D layer that is in direct contact with the magnetic material ${ }^{53,54}$. The latter opens opportunities for the creation of synthetic spin-valve structures based on non-magnetic $2 \mathrm{D}$ materials ${ }^{55}$.

Recently Asshoff et al. ${ }^{23}$ were able to investigate pristine (clean, unoxidized) FM/Gr interfaces, adapting the process developed by $\mathrm{Li}$ and coworkers ${ }^{21}$ of directly depositing FM on either side of a suspended graphene sheet. The authors have also annealed the samples to achieve conformal Van der Waals interfaces, and decouple any possible hybridization between FM and Gr. Crucially, this process allowed the study of doping and proximity induced exchange splitting in graphene upon the spin polarization. In a stack of FM/nGr/FM, each of these graphene layers experienced doping and magnetic proximity effect from the FM electrode in direct contact with their corresponding interface. Depending on the chemical nature of the FM layer, graphene can get $\mathrm{p}$-doped or $\mathrm{n}$-doped, resulting respectively in spin-down or spin-up spin-polarized graphene interface(s). The doping level of the graphene layer can also be tuned by applying a bias voltage between the source and drain electrodes of the MTJ device, playing the same role as a gate electrode (Fig. 4). While the proximity effect introduces an extrinsic spin polarization $\sim 10 \%$ due to exchange splitting of $\sim 6 \mathrm{meV}$, the resulting chemical potential shift from bias voltage allows going from positive to negative spin polarization, with however one decade lower MR amplitude when compared to previously discussed Ni/CVD-Gr/oxide barrier based $\mathrm{MTJ}^{18,26,28}$. Beyond the magnetic proximity observed for conducting graphene, we will discuss in the following section how the proximity effect and Van der Waals gap between magnets and insulating 2D crystals could lead to chemisorption and physisorption based distinct spinterfaces.

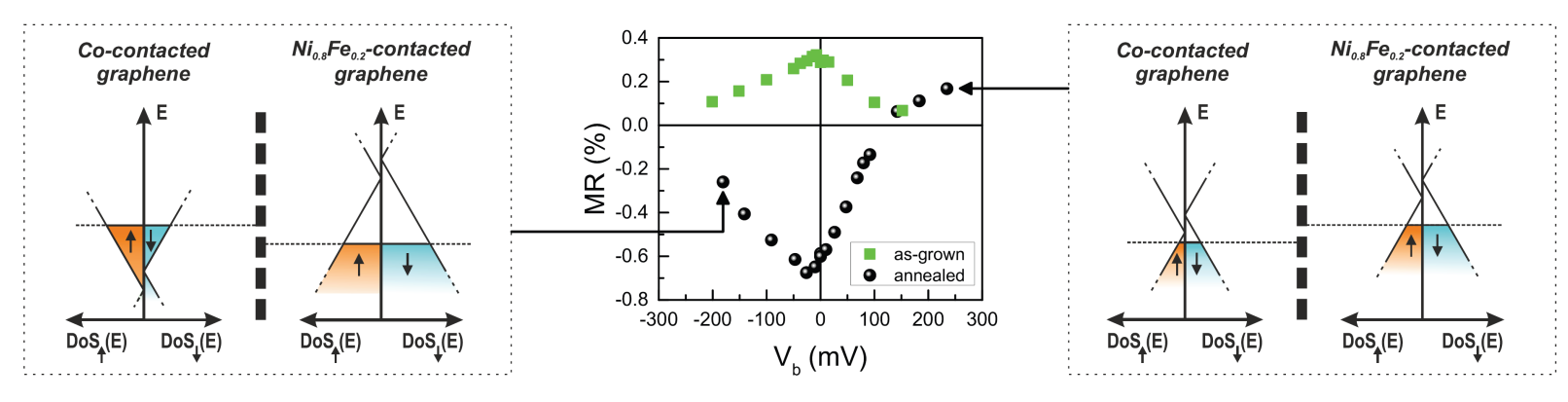

Figure 4. Magnetic proximity effect in annealed Gr/FM interface. Depending on both the doping level and proximity exchange splitting induced into the graphene barrier by the adjacent ferromagnetic layer, the graphene/ferromagnet electrodes can lead to either negative (left panel) or positive (right panel) spin polarization. Reproduced with permission from Asshoff, P. U. et al., 2D Mater. 4, 031004 (2017). 


\section{Ferromagnet-2D interfaces beyond graphene}

\section{A. Ferromagnet-2D insulator interfaces}

Despite the unique spin filtering properties of multilayered graphene in ferromagnetic junctions, its ability to obtain a desirable resistance-area product (RA) remained limited to a few $\Omega . \mu m^{2}$ in thermally improved interfaces. To obtain high magneto-resistance for applications, achieving controlled RA reaching up to few k $\Omega$ $\mu \mathrm{m}^{2}$ is necessary ${ }^{56,57}$. In addition, for exploring planar spin circuits utilizing graphene or semiconductors, it is necessary to have interfaces that provide optimum resistance to overcome the conductivity mismatch problem. While conventional metal oxides such as $\mathrm{MgO}, \mathrm{TiO}_{2}$, or $\mathrm{Al}_{2} \mathrm{O}_{3}$ have been employed to address this problem, the 2D material Van der Waals family has promising substitute insulating crystals such as $h B N$, fluorographene, and hydrogenated graphene. In the past few years, there have been demonstrations of how such crystals improve spin injection and their suitability as atomically thin spacers for magnetic tunnel junctions. Here, we will focus our discussion on hexagonal boron nitride, a standard 2D insulator that is widely used in 2D nanoelectronic and spintronic devices. Hexagonal boron nitride is structurally equivalent to graphene, yet a bandgap of $\sim 6 \mathrm{eV}$ makes it an insulator, ideal for applications as a substrate, gate dielectric, and a tunnel barrier in graphene electronic devices. All these multiple uses of hBN make it a versatile material for combining graphene/2D electronics and spintronics. In the specific context of spintronics, with no interface states, hBN shows real promise for the ideal role of an atomically thin insulator for tunnel barrier applications ${ }^{58}$ in both magnetic tunnel junctions, as well as lateral spintronic applications ${ }^{59-62}$. In addition to offering a suitable interface resistance due to its insulating nature, it also shows novel spin filtering properties when combined with ferromagnets that leads to enhanced values of spin polarization and magnetoresistance. Theoretical calculations ${ }^{63,64}$ have indicated such possibility and increased spin polarization of ferromagnetic interfaces with hBN multi-layers, due to the spatial enhancement of spin accumulation density within the layers of hBN. Encouraged by the initial findings, both exfoliated $\mathrm{hBN}$ and large-scale chemical vapor deposited hBN have been employed in MTJs. A list of recent experimental findings using hBN as spacers between ferromagnetic electrodes is displayed in Table-II and reveals a maximum TMR 50\% with a tunnel spin polarization $60-80 \%$ for Colh-BN interfaces at $1.4 \mathrm{~K}$ and $\mathrm{P} \sim 7 \%$ at $300 \mathrm{~K}^{23}$. Theoretical reports predict preferential spin filtering at the hBN|ferromagnet junctions, leading to spin polarization $\sim 100 \%$ with an increased number of layers $^{64}$. Here the hetero-junctions are considered to have perfect lattice matching and hybridization of the orbitals. However, experimental conditions need not attain such perfect conditions. Overall, there is a consensus on the coupling between hBN and ferromagnets as the prime factor that influences polarization. In addition, bias dependent sign change in MR has also been observed in hBN based devices encompassing additional layers of graphene ${ }^{65,66}$. The change in sign of polarization in $\mathrm{Co} / \mathrm{Gr}$ interfaces has been attributed to electric-field assisted tuning of the Fermi level and the proximity induced exchange spin splitting in graphene ${ }^{23}$. Bias assisted inversion has also been observed in hBN junctions with ferromagnets, as reported recently ${ }^{67}$ (shown in Fig. $5)$, implying a more subtle role of coupling between hBN and ferromagnets. Very recently, the role of

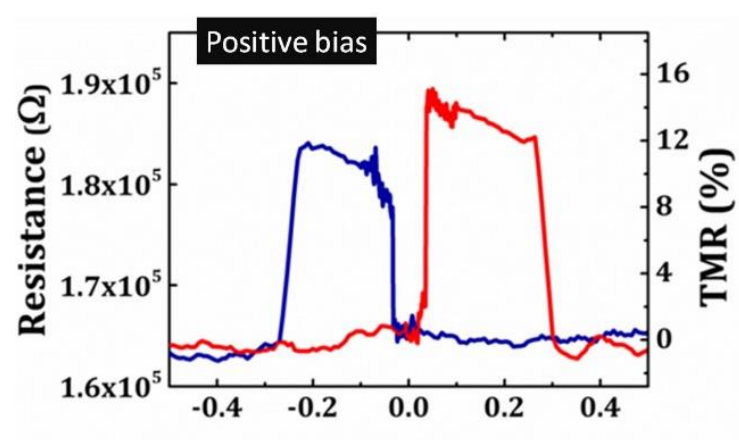

Magnetic field applied ( $\mathrm{T}$ )

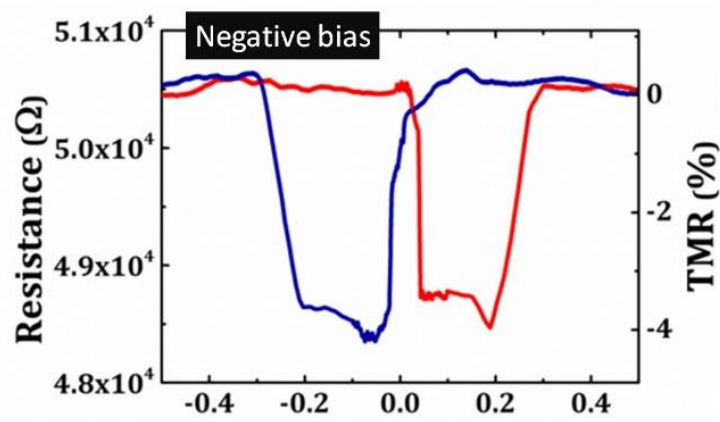

Magnetic field applied (T)

Figure 5. Bias assisted inversion has been observed in Co $|\mathrm{hBN}| \mathrm{hBN} \mid \mathrm{Co}$ junctions ${ }^{67}$. Adapted with permission from Piquemal-Banci, M. et al., ACS Nano 12, 4712 (วn18)

point defects in h-BN interfaces with ferromagnets has been investigated ${ }^{68}$, showing voltage controlled specific enhancements in magnetoresistance, suggesting the role of resonant tunneling through magnetic defects and their proximity exchange coupling with the ferromagnetic electrodes. 
Table 2: Experiments involving hBN spacer in MTJs

\begin{tabular}{|c|c|c|c|c|c|}
\hline MTJ structure & Ref. & Layer thickness & MR (\%) (Temperature) & $\begin{array}{l}\text { Polarization } \\
\text { (Jullier's } \\
\text { formula) }\end{array}$ & $\begin{array}{c}\text { 2D layer Growth } \\
\text { Method }\end{array}$ \\
\hline NiFe|h-BN |Co & \multirow{2}{*}{69} & Monolayer & $0.3 \%(300 \mathrm{~K})$ & $4 \%$ & \multirow{2}{*}{$\begin{array}{l}\text { CVD h-BN } \\
\text { Wet transfer in } \\
\text { isopropyl alcohol }\end{array}$} \\
\hline Co|h-BN |Co & & Monolayer & $0.5 \%$ (300 K) & $5 \%$ & \\
\hline Colh-BN|Fe & 70 & Monolayer & $6 \%(1.5 \mathrm{~K})$ & $17 \%$ & $\begin{array}{l}\text { CVD directly grown } \\
\text { on Fe }\end{array}$ \\
\hline ColhBN/NiFe & 23 & Bilayer & $\begin{array}{l}1 \%(10 \mathrm{~K}) \\
0.6 \%(300 \mathrm{~K})\end{array}$ & $7 \%$ & $\begin{array}{l}\text { Exfoliated hBN, post } \\
\text { transfer annealing }\end{array}$ \\
\hline Co|Gr-hBN|NiFe & 23 & $\begin{array}{l}\text { Monolayer Gr, } \\
\text { bilayer hBN }\end{array}$ & $\begin{array}{l}\sim 1 \%(10 \mathrm{~K}) \\
\text { bias induced inversion } \\
\text { in pol. }\end{array}$ & $7 \%$ & $\begin{array}{l}\text { Exfoliated Gr, hBN, } \\
\text { post-transfer } \\
\text { annealing }\end{array}$ \\
\hline Co|h-BN|h-BN|Co & 67 & $\begin{array}{l}\text { Two layers of } \\
\text { hBN }\end{array}$ & $\begin{array}{l}12 \%(1.4 \mathrm{~K}) \\
\text { bias induced inversion } \\
\text { in pol. }\end{array}$ & $25-30 \%$ & $\begin{array}{l}\text { Colh-BN is directly } \\
\text { grown, } 2^{\text {nd }} \text { layer is } \\
\text { transferred by wet }\end{array}$ \\
\hline Co|h-BN|Fe & & $\begin{array}{l}\text { Monolayer } \\
\text { hBN }\end{array}$ & $50 \%(1.4 \mathrm{~K})$ & $60-80 \%$ & $\begin{array}{l}\text { transfer techniques } \\
\text { CVD }\end{array}$ \\
\hline $\mathrm{Ni}|\mathrm{hBN}| \mathrm{Ni}$ & 66 & - & $0.83 \%(4.2 \mathrm{~K})$ & $6.4 \%$ & CVD Gr transferred \\
\hline & & & $0.54 \%(300 \mathrm{~K})$ & $5.2 \%$ & \\
\hline $\mathrm{Ni}|\mathrm{Gr}| \mathrm{hBN} \mid \mathrm{Ni}$ & 66 & $\begin{array}{l}\text { Monolayer } \\
\text { graphene }\end{array}$ & $\begin{array}{l}-1.83 \%(4.2 K) \\
-0.56 \%(300 K)\end{array}$ & $\begin{array}{l}-9.52 \% \\
-5.28 \%\end{array}$ & $\begin{array}{l}\text { CVD materials } \\
\text { transferred }\end{array}$ \\
\hline Colh-BN | NiFe & 68 & 2-4-layer hBN & $\begin{array}{l}2.2-3.6 \%(10 \mathrm{~K}) \\
\text { Voltage controlled MR } \\
\text { \& resonant tunneling }\end{array}$ & $10-13 \%$ & exfoliated \\
\hline
\end{tabular}

\section{Chemisorption vs. physisorption}

As described in the previous section, the coupling of ferromagnet with graphene plays a significant role in determining the spin polarization, as well as doping in graphene. It has been reported that the process of fabrication could influence the lattice overlap at the ferromagnet-hBN interface, which could lead to chemisorption for a commensurate lattice-matched configuration, or a more Van der Waals interaction for physisorption of hBN over ferromagnets ${ }^{67}$. In fact, the coupling between $\mathrm{hBN}$ and ferromagnet dramatically varies from commensurate configuration (with the distance between $\mathrm{hBN}$ and Co $2.1 \AA$ ) to incommensurate configuration for $1 \AA$ additional gap between hBN and Co. While hBN transferred on ferromagnetic electrodes may not always lead to a commensurate interface, a directly grown hBN on ferromagnetic Co is seen to lead to commensurate conformation. The commensurate structure results in interface hybridization showing a bias dependent polarization inversion ${ }^{67}$. A schematic showing the distinct effects is displayed in Fig. 6.

Apart from MTJs, the spin signal has also been observed in planar spin valves, for example in systems where the spin injection is performed through the FM-hBN tunnel junction into graphene. In these systems, the hBN thickness dependent enhancement in spin polarization is also reported ${ }^{65}$, with bias dependent sign change of the spin polarization. A spatial variation in interface conditions or a mixture of the commensurate and incommensurate configurations and partial dominance of one over the other could make a significant impact on such experimental observations. In addition, the entire structure of the composite barrier in FM-hBN-Gr can show spin selectivity ${ }^{64}$, while the bias induced doping of graphene could further play a key role in the observed characteristic bias dependent inversion of polarization. 


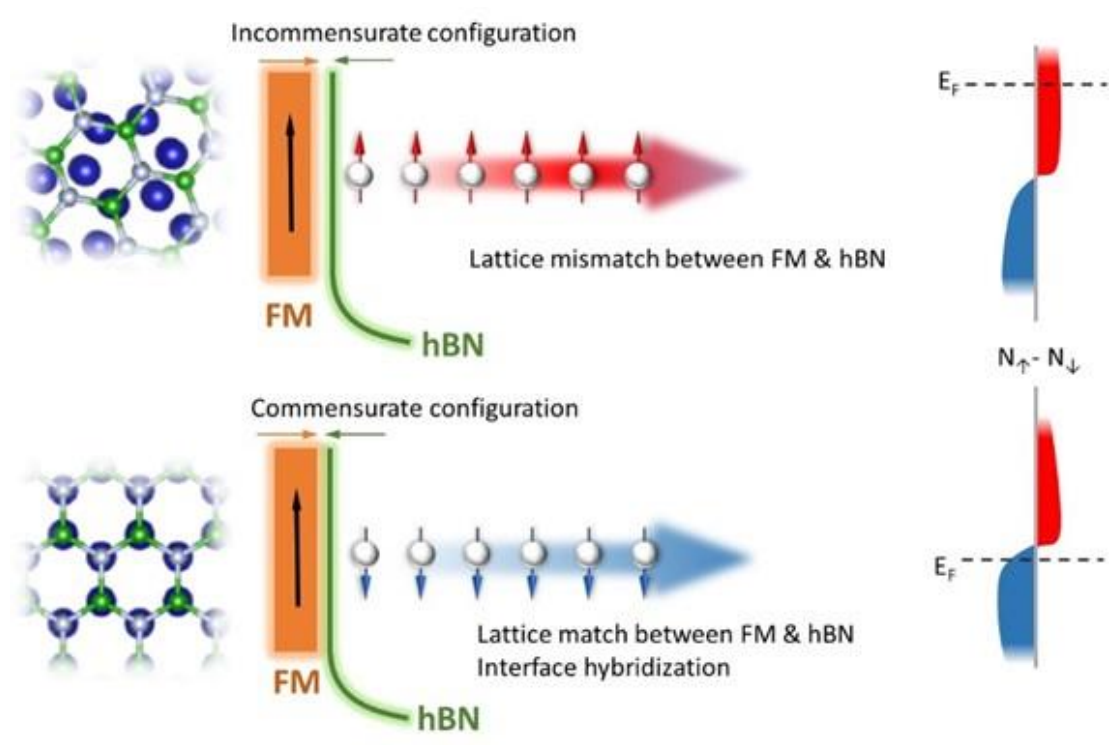

Figure 6. A depiction of preferential spin transmission across incommensurate and commensurate interfaces ${ }^{67}$ that leads to distinct inversion of magnetoresistance.

\section{B. Ferromagnet-2D semiconductor junctions}

Having a bandgap in a graphene-like material is essential for semiconductor electronics. This has led to the exploration of 2D semiconducting crystals, in particular, the class of transition metal dichalcogenides (TMD), which has shown huge potential for optoelectronic and nanoelectronic applications. The $T M D s\left(M X_{2}, M=\right.$ Transition metal, $X=$ Chalcogen) offer a large number of crystals with diverse electronic properties ranging from metals to semiconductors, superconductors, and magnets. Furthermore, the tunable bandgap changes from indirect to direct with a reduction of the number of layers, providing extra knobs for engineering. Investigation of TMDs as spacers in magnetic tunnel junctions is necessary considering the potential of integration of optoelectronics and nanoelectronic phenomena with spintronic capabilities.

MTJ structures made with TMDs have been studied through experimental and theoretical routes in both current-in-plane (CIP) and current-perpendicular-to-plane (CPP) geometries, which are tabulated in Table 3. Structures involving $\mathrm{MoS}_{2}{ }^{71-79}, \mathrm{WS}_{2}{ }^{80,81}, \mathrm{WSe}_{2}{ }^{82}$, and $\mathrm{WTe}_{2}{ }^{83}$ were studied with ferromagnetic (FM) electrodes made of Permalloy $\left(\mathrm{Ni}_{80} \mathrm{Fe}_{20}\right)^{82,84}, \mathrm{Fe}^{71,76}, \mathrm{Co}^{74,75,81}, \mathrm{Ni}^{75}$, half-metallic $\mathrm{CrO}_{2}{ }^{72}, \mathrm{La}_{0.7} \mathrm{Sr}_{0.3} \mathrm{MnO}_{3}{ }^{73}, \mathrm{Fe}_{3} \mathrm{O}_{4}{ }^{78}$, and $\mathrm{Fe}_{3} \mathrm{Si}^{77}$. Ab-initio transport calculations involving non-equilibrium Green's function formalism (NEGF) have predicted significant spin injection and enhanced MR in most of the cases. Commonly, in the CPP geometry, the $M R$ increases with an increase in the TMD layer thickness which gets saturated (but robust) at a higher thickness as long as it stays within the tunneling limit. The thinner junctions made with single or bilayer of TMD are mostly metallic due to the strong coupling (hybridization) between the FM and interfacial TM atoms, which becomes insulating at larger thicknesses. For such thin junctions, MR is entirely dominated by the electronic structures of the FM electrodes, and induced magnetization is thickness-dependent in the TM atoms located near FM due to the strong overlapping of their electronic states. Overall, a high predicted optimistic MR up to $800 \%$ in theoretical investigations, together with the electronic nature of such crystals has made a great compelling case for experimental investigations in this direction. However, experimental results on such systems revealed results that are yet to match high theoretical expectations. In general, it has been observed that based on the method of preparation of the TMD layer, the MR ratio varied significantly. Using a mechanically exfoliated $T M D^{81,82}$ layer, spin valve effect was observed from room temperature with a maximum $\mathrm{MR}$ at $4.2 \mathrm{~K}$ at which the layer became metallic. Observation of a negative $\mathrm{MR}$ in $\mathrm{Py} / \mathrm{WSe}_{2} / \mathrm{Py}^{82}$ was reported, which could likely be due to asymmetric interface intrinsic to the different condition under which the interfaces were formed. Using PVD grown $W_{2}$ single crystal nanoflakes, a temperature-controlled negative to positive crossover $^{80}$ in the MR was reported around $90 \mathrm{~K}$. This was attributed to be originating from a crossover between weak-localization and weak-antilocalization and disorder-induced scattering in WSe $\mathrm{S}_{2}$. Such crossover was also reported in $\mathrm{WTe}_{2}$ (CIP configuration) ${ }^{83}$ with a change of the magnetic field direction. A negative MR was observed when the applied magnetic field and electric field are parallel to each other and the strong electric field induced spin-orbit coupling is responsible behind this crossover. The highest MR ( 120\%) is lower than the value reported for bulk value and the difference could be accounted to the unequal numbers of 
electrons and holes in the material.

Table 3: MR behavior of various MTJ structures with TMD spacer layers

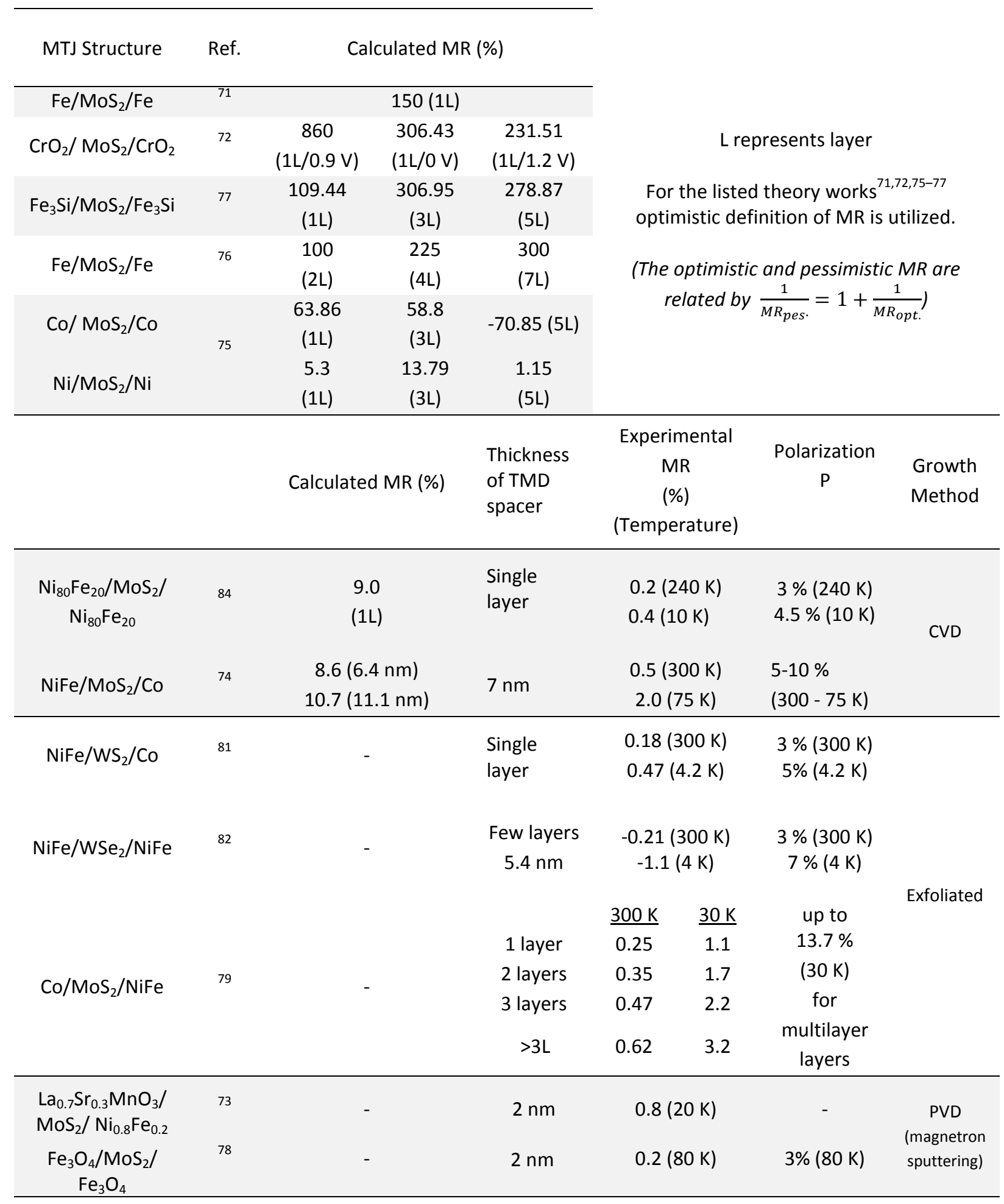

Several studies have been reported on $\mathrm{MoS}_{2}$ based tunnel junctions with various FM electrodes ${ }^{73,84,79}$. It was observed that capping the FM electrodes prevented oxidation and enhanced the MR ratio. Commonly the $\mathrm{MoS}_{2}$ layer was found to be polycrystalline, and a single crystalline phase is expected to yield better MR behavior. Using different FM layers on two sides of $\mathrm{MoS}_{2}$, an improvement in the MR was observed in $\mathrm{Py} / \mathrm{AlO}_{\mathrm{x}} / \mathrm{MoS}_{2} / \mathrm{Co}^{72}$ and $\mathrm{Co} / \mathrm{MoS}_{2} / \mathrm{NiFe}^{79}$. Typically, the observed MR corresponds to spin polarization of 5-15\%, which is lower than the theoretically predicted values, and the difference is caused by the lattice mismatch between the two layers and the presence of defects leading to enhanced scattering. To improve the MR performance, half-metallic FM 
electrodes were used in several studies as they can offer almost $100 \%$ spin polarization at the interface and better spin injection into the TMD layer as also supported theoretically ${ }^{77}$. However, the experimental MR value was observed to be lower than the theoretical predictions, which was attributed to the lack of transparency in the FM/TMD interfaces. Theoretically very high MR values were predicted for $\mathrm{MoS}_{2}$ with $\mathrm{CrO}_{2}(\mathrm{MR} \sim 860 \%, \mathrm{CIP}$ configuration $^{72}$ ), and Heusler alloy $\mathrm{Fe}_{3} \mathrm{Si}$ (MR > 300\% and Spin Injection efficiency (SIE) of $80 \%$ ) which were found to be robust with an increase in the applied bias ${ }^{77}$. However, the experimental demonstration of such values remains a challenge.

The MR signal, in general, is found to increase with a reduction of temperature due to the decrease in scattering from magnetic impurities, the presence of surface states, inelastic scattering with phonons, and thermal broadening of the electronic energy levels in the FM electrodes. The temperature dependence of the $M R$ ratio follows the same trend of the surface magnetization, which can be described using Bloch's $T^{3 / 2}$ law: $P(T)=P(0)\left[1-\alpha T^{3 / 2}\right]$, where $P(T)$ is the spin polarization at temperature $T$ and $\alpha$ is a constant. The value of $\alpha$ determines a fast/slow fall of the MR at a higher temperature, which is associated with the Curie temperature $\left(T_{c}\right)$ of the FM and the barrier height. The MR signal was found to increase with a reduction of the bias current. Such behavior could be attributed to the presence of localized spin excitations, magnons and localized trapped states in the interlayer. Similarly, band bending, magnon excitation and appearance of interface states contribute towards the decrease in MR at higher applied bias ${ }^{85}$.

It is widely observed that theoretical calculations predict a very high MR and significantly large spin polarization for an FM/TMD interface, whereas experimentally they show lower values. There are several reasons behind this. Firstly, the nature of the layer considered is ideal in theory: it is unstrained, without defects and distortions; however, experimentally obtained layers are far from perfect. In most of the cases, the layers are polycrystalline and not entirely uniform throughout the junction area. The electronic properties of $\mathrm{MoS}_{2}$ were found to be strongly dependent on the number of layers, which for monolayer is metallic, but insulating at higher thicknesses. The nature of the tunnel barrier also changes with thickness, being more insulating for a thicker layer. This is different from the single-crystalline nature of the layer with perfect tunnel barriers considered in theory. In the reports with giant MR signals, the size of the supercell used is not always of similar size as that used in the experiments. During the wet transfer of the TMD, surface adatoms, contaminants, or cavities can't be removed completely, and they play a role in the interface transparency. Depending on the FM/TMD combination and method of preparation of the TMD layer, some interfacial strain is inevitable, and this can hinder the spin propagation. Some FM electrodes are prone to oxidation, and capping them with a protective layer (thin oxide layer) is useful to improve the performance. Conductivity mismatch between the electrode and the tunneling layer can also be a factor to consider, and an intermediate buffer layer can help to improve the performance. Unlike Graphene and h-BN, spin-orbit coupling is significantly large in TMDs, which can cause additional spin-flip scattering and reduction of MR ratio. In an ideal MTJ, the carrier motion should be in the ballistic regime while in experiments, it is found to be mostly diffusive due to the imperfections and inelastic scattering from the presence of impurities affecting the tunneling performance.

Based on the available results, a recipe for improvement of the MR could be suggested. The choice of the electrode material is very crucial. A stronger FM (preferably half-metallic) with proper oxidation protection is necessary. A thin oxide-insulating layer with better lattice matching between the FM and TMD can help in stabilizing the structure and a conductivity match. An asymmetric FM combination can offer easy switching of the FM blocks at different magnetic fields due to different coercivity as used by Dankert et al. ${ }^{74}$ and Khan et al. ${ }^{79}$ with the latter observing the highest MR ( 3.2\%) within the available reports. It is expected that a similar structure will result in a higher MR at much lower temperatures. If the anisotropy of the TMD plays a role, then an angular dependent MR measurement (varying the angle between current and magnetic field) would help in identifying the preferred direction of the applied field to reach the maximum MR. It is generally observed that the MR ratio improves with thicker layers of single-crystalline nature. Van der Waal's heterostructure of TMDs can be tested for this purpose. Special care should be taken during the wet transfer process of the CVD grown or mechanically exfoliated TMD layer and while dealing with any interface. The lack of transparency in the quality of the FM/TMD interfaces is a possible suspect behind the lower MR ratio. In situ preparation of the entire heterostructure in a controlled environment could circumvent most of these problems. Despite such care, the theoretically predicted MR values are usually the maximum upper limit, and experimental results are expected to provide smaller values. The Ab-initio calculations typically provide results under equilibrium conditions, and very low-temperature measurements are essential for comparison. However, no experimental report is available below $4 \mathrm{~K}$. Therefore, there are still opportunities for theoretical studies to incorporate 
practical factors into their model and see the changes in MR performance.

\section{All 2D magnetic tunnel junctions}

So far, we have been discussing 3D ferromagnetic junctions with 2D materials. The use of ferromagnet ultrathin film in magnetic tunnel junction is now explored for almost four decades, ${ }^{37,86-88}$, which are mainly fabricated by physical growth methods such as molecular beam epitaxy, sputtering, or pulsed laser deposition ${ }^{89-91}$. Optimizations and research with such materials have led us to present-day highly efficient data storage and magneto-sensing technologies. However, research showed several issues relating to disorder present in the electrodes, barrier and electrode-barrier interface, interdiffusion ${ }^{92}$ that affect spin polarization of the FM, and its interface with the tunnel barrier. Interface roughness results in local variations of the barrier thickness, local magnetic fringe field, and inhomogeneous electronic configuration and coupling at the interfaces that may strongly affect the spin-dependent tunneling conductance ${ }^{93-96}$. Impurities, vacancies, and defects present in the barrier or at the interface of covalently bonded materials can trigger inelastic assisted tunneling mechanisms ${ }^{97-101}$. Spin transport across tunnel junctions can also be modified by the presence of crystal grains, stacking faults, which are sensitive to details of atomic sequence at the interface ${ }^{2,3,102,103}$. These challenges can be naturally bypassed by employing a layer by layer assembly of magnetic and non-magnetic 2D materials. Here, the recent discovery of two-dimensional magnetism (up to single layers of atoms) provides an innovative landscape for magnetic tunnel junctions, via the formation of pristine interfaces with on-demand structural integrity due to their Van der Waals nature. Hence such materials and their van der Waals heterostructures offer several new possibilities. For example, 2D materials with different crystal structures and lattice constants can be stacked into heterostructures, without the structural and electronic detrimental effect seen in conventional epitaxial thin films ${ }^{104,105}$. Since the interlayer interaction is of the van der Waals kind, the 2D materials forming FM/Insulator/FM device ${ }^{106,107}$ architectures can stay electronically decoupled, circumventing complex covalent hybridization at interfaces and interface states issues with traditional evaporated metaloxide interfaces $^{2,3,103}$. This implies that van der Waal's magnetic layers introduce new concepts of spin filtering beyond the usual spin injection/detection schemes while exploiting only the change of magnetic ordering into the lamellar structure ${ }^{108-110}$. Here we briefly introduce the emergent $2 \mathrm{D}$ magnetic systems and new exotic applications of relevance to the area constructing all 2D magnetic tunnel junctions.

\section{Magnetism in two-dimensional materials}

Atomic magnetic moment, arising out of unpaired electrons, is a necessary ingredient of magnetism. In a lattice, adjacent atomic moment or spins are coupled through a quantum mechanical exchange interaction result in magnetic properties of materials. Magnetism depends on the lattice dimensionality or crystal structure, as well as the spin-dimensionality of the system. For a 2D layer of magnetic moments, Fig. 7 shows three common configurations of spin dimensionality, where the atomic spins could be Ising (pointing up or down) type, or oriented in a plane (XY model) or more like a Heisenberg (XYZ model) kind. Theoretically, the existence of magnetism in 2D dimensions for spins with continuous symmetry is excluded by the MerminWagner theorem ${ }^{111}$. However, anisotropy can enable sustainable long-range magnetic ordering, which has been experimentally observed in several magnetic 2D materials recently. It is worth noting that 2D Ising kind of behavior has been reported in neutron scattering experiments in layered materials ${ }^{112}$ much earlier. The existence of magnetism down to monolayers in several magnetic 2D materials has been established very recently; some of these crystals are presented in Table IV.
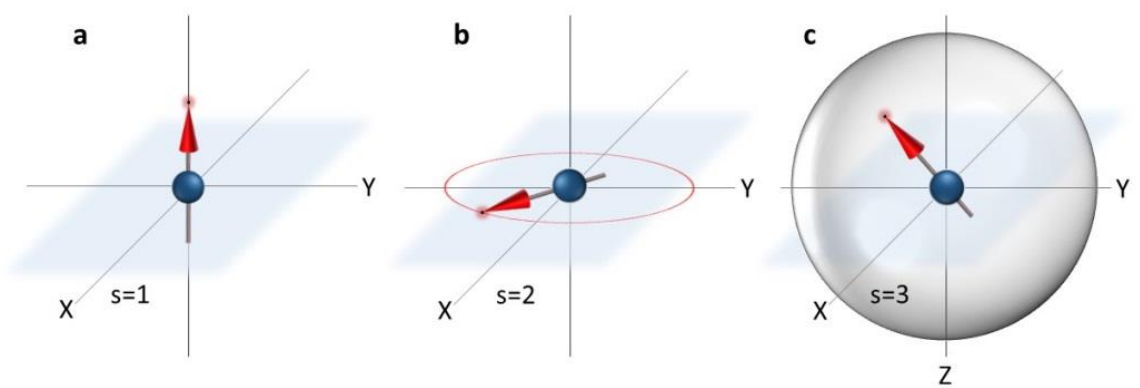

Figure 7. Spin dimensionality S (a) Ising configuration with spin pointing up or down along Z-axis. (b) XY-model, where spins are free to orient towards any point (shown by the red dot) on the circle in the XY plane. (c) Heisenberg kind, the sphere is drawn to show that spins can orient to any point (indicated by the red dot) on the sphere. 
Intrinsic magnetic order in 2D layered crystals was discovered in early 2017 in two unique systems at low temperatures ${ }^{113,114}$. One of these crystals, $\mathrm{Cr}_{2} \mathrm{Ge}_{2} \mathrm{Te}_{6}$ remarkably showed ferromagnetism down to the bilayer limit, with a suppression of the Curie temperature, where the ferromagnetic to paramagnetic phase transition occurs, as observed in low-dimensional magnets ${ }^{113}$. Concurrently, the ferromagnetic order was also shown to exist in monolayer $\mathrm{Crl}_{3}$ up to $45 \mathrm{~K}$. Moreover, bilayer $\mathrm{Crl}_{3}$ showed no net magnetization due to the antiferromagnetic coupling between the two layers. In addition to these low-temperature demonstrations, recently stable ferromagnetic order has been reported in $\mathrm{VSe}_{2}$ crystals on graphite and $\mathrm{MoSe}_{2}$ at room temperature ${ }^{115}$. Several such materials have been theoretically predicted to possess ferromagnetism, including $\mathrm{VSe}_{2}{ }^{116}$. Primarily, this allows for the understanding of spin interactions and phase transitions in two dimensions.

Furthermore, due to their reduced dimensionality, they present a direct response to external stimuli such as mechanical strain, electric field effects, and optical excitation. The presence of magnetism in monolayers also suggests the tunability of exchange interaction and transition temperatures upon the influence of stimuli. At the same time, spin linked Hamiltonians are expected to explore quantum phase changes, for instance, Kosterlitz-Thouless transition in $\mathrm{XY}$ configurations. As described in the next section, new interfaces with other novel materials could be employed to tune properties much more effectively. Propelled by these possibilities, the $2 \mathrm{D}$ magnetism rose to prominence with several teams demonstrating magnetism in new crystals ${ }^{117}$, electric field control of magnetism ${ }^{118,119}$, observing huge values of up to $\sim 104 \%$ magnetoresistance ${ }^{108,120}$ and tunnel spin filtering through $\mathrm{CrBr}_{3}$ originating from spin-magnon activated inelastic tunneling ${ }^{121}$. The latter reported the first observation of proximity induced magnetoresistance in graphene from a 2D ferromagnet ${ }^{121}$.

Table-IV: Some experimentally characterized 2D magnetic systems.

\begin{tabular}{|c|c|c|c|c|c|}
\hline 2D magnet & Ref. & Layer thickness & Tc & Easy axis & Electrical property \\
\hline $\mathrm{Cr}_{2} \mathrm{Ge}_{2} \mathrm{Te}_{6}(\mathrm{CGT})$ & 113 & $\begin{array}{l}\text { Bilayer } \\
\text { Few layer }\end{array}$ & $\begin{array}{l}30 \mathrm{~K} \\
61 \mathrm{~K}\end{array}$ & Out of plane & Insulator \\
\hline $\mathrm{Crl}_{3}$ & 114 & $\begin{array}{l}\quad \text { Monolayer } \\
\text { (ferromagnet for } \\
\text { odd number of } \\
\text { layers, anti- } \\
\text { ferromagnet for } \\
\text { even number of } \\
\text { layers) }\end{array}$ & $\begin{array}{l}45 \mathrm{~K} \text { (monolayer) } \\
\text { (Bulk form 65 K) }\end{array}$ & Out of plane & Insulator \\
\hline $\mathrm{Fe}_{3} \mathrm{GeTe}_{2}(\mathrm{FGT})$ & 122 & $\begin{array}{l}\text { 5-25 nm } \\
\text { Monolayer }\end{array}$ & $\begin{array}{c}155-200 \mathrm{~K} \\
\text { (bulk Tc } 207 \mathrm{~K} \text { ) } \\
130 \mathrm{~K}\end{array}$ & Out of plane & Metallic \\
\hline $\mathrm{VSe}_{2}$ & 115 & Monolayer & $\begin{array}{l}>300 \mathrm{~K} \\
\text { (paramagnetic in } \\
\text { bulk form) }\end{array}$ & In-plane & $\begin{array}{c}\text { Metallic in } 1 T \text { crystal } \\
\text { structure form }\end{array}$ \\
\hline $\mathrm{CrSi} \mathrm{Te}_{3}$ & 123 & Few layers $(7 \mathrm{~nm})$ & $\begin{array}{c}\text { 80-120 K } \\
\text { (observed from } \\
\text { resistivity) } \\
\text { (Bulk Tc } \sim 33 \mathrm{~K} \text { ) }\end{array}$ & Out of plane & semiconductor \\
\hline
\end{tabular}


A variety of 2D ferromagnets have emerged, opening new prospects for studying junctions with graphene and hexagonal boron nitride that have so far been studied with conventional ferromagnetic materials. Recently Z. Wang et al. demonstrated a magnetic tunnel junction in $\mathrm{Fe}_{3} \mathrm{GeTe}_{2} / \mathrm{hBN} / \mathrm{Fe}_{3} \mathrm{GeTe}_{2}$ Van der Waals heterostructures ${ }^{107}$ (Fig. 8). Such structures allow for probing sharp interfaces. The authors obtained an optimistic magnetoresistance $160 \%$ at low temperature, yielding a spin polarization 66\% (83\% for the majority and $17 \%$ for minority spins). Their measurements of spin valves and temperature dependence of spin polarization corroborated precisely to the anomalous Hall conductivity results, indicating an interface nature quite similar to the bulk ferromagnet. The report also shows that, with increasing temperature, the evolution of the spin polarization extracted from the tunneling magnetoresistance is proportional to the temperature dependence of the magnetization obtained from the analysis of the anomalous Hall conductivity. This suggests that all 2D material based MTJs provide new means for exploring pristine interfaces that are not affected by heterostructure formation, providing a sharp transition in the 2D material layers that is not possible by the growth of FM electrodes. The perpendicular nature of magnetization to the crystal planes result in relatively larger magnetic switching fields. However, crystals like $\mathrm{VSe}_{2}$ showed lower switching fields that show potential for low field operation. This area is still in its infancy and presents fresh opportunities to explore magnetism in two dimensions, 2D magnetic tunnel junctions, and spinterfaces with unique lattice configurations.

\section{Exotic ferromagnetic (eFM) heterostructures for tunable junctions}

Often in normal ferromagnet-nonmagnet junctions in spintronic devices, it is not only challenging to obtain smooth interfaces, but it is also not feasible to tune the properties of the ferromagnetic layer dynamically. This restricts the FM-NM junctions to specific properties based on the interface. On the other hand, unlike topdown fabricated ferromagnetic thin films, the 2D magnetic crystals have made the unprecedented realization of true magnetism in two dimensions, down to the level of crystal monolayers. Realizing magnetism in atomically thin layers implies multiple possibilities for enhanced control using electrostatic doping, chemical doping, optically assisted modulation, and proximity induced effects. Such opportunities could lead to designing exotic heterostructures of 2D ferromagnets (instead of 3D ferromagnets) that enable additional control of interface spin conductance. The band structure of atomically-thin 2D materials is greatly influenced by the electronic nature of adjacent materials. For instance, heterostructures of bilayer $\mathrm{Crl}_{3}$ with graphene show tunable magnetism, including the possibility of antiferromagnetic to ferromagnetic switching by electrostatic doping ${ }^{119,124,125}$. The creation of new spinterfaces of 2D ferromagnets with 2D semiconductors ${ }^{126}$ has demonstrated hybrid spin and valley polarization capabilities, prospective for the integration of information processing and memory storage circuits. Beyond 2D materials, novel 3D Topological insulators (TI) exhibit surface conducting states protected by time-reversal symmetry, with spin-momentum locking. TI heterostructures involving 2D FM, therefore, attain new capabilities, like the presence of large anomalous Hall effect $^{127}$. In such heterostructures with $\mathrm{TI}$, the proximity coupling with $\mathrm{FM}$ can lead to enhanced interface magnetism $^{128}$, enhancing the operation temperature. The TI|FM heterostructures are favorable for engineering magnon dispersion, magnetocrystalline anisotropy. In addition to proximity induced modifications in heterostructures, stacking order and interlayer arrangement in a multilayer 2D magnet itself could be employed to make striking changes in magnetic properties ${ }^{129-131}$. In particular, stacking order can be tuned by applying pressure as well as introducing disorder. For example, lamellar magnetic semiconductor $\mathrm{crystal}^{\mathrm{RuCl}} \mathrm{Cl}_{3}$ shows that adding turbostratic disorder (slipping of atomic layers) in a stack of $\mathrm{RuCl}_{3}$ nanosheets allows for 
tuning the interlayer interaction and modifying the 2D layers stack magnetic poperties ${ }^{132}$. A recent report on a few $\mathrm{nm}$ thick $2 \mathrm{D} \mathrm{CrCl}_{3}$ crystal showed a ten-fold enhancement of the interlayer exchange coupling compared to bulk $\mathrm{CrCl}_{3}$ due to the intrinsically different stacking order ${ }^{133}$. Change of interlayer stacking order also results in a shift in the magnetic state from $\mathrm{FM}$ to $\mathrm{AFM}$, as observed in $\mathrm{Crl}_{3}$ while using hydrostatic pressure to tune the interlayer distance and arrangment ${ }^{134}$. Similarly, distinct interlayer magnetism was also observed in bilayer $\mathrm{CrBr}_{3}$ by spin-polarized scanning tunneling spectroscopy, where measurements revealed antiferromagnetic coupling in rhombohedral stacking while ferromagnetic coupling in monoclinic layer stacking ${ }^{135}$. In addition to their core fundamental importance, these results indicate strategies to modulate the critical switching fields and the magnetic phase by changing the interlayer coupling, thereby presenting exotic aspects for building novel MTJs. Therefore, stacking-dependent properties like orbital hybridization and strain emerge as new control-knobs to design exotic 2D ferromagnets (eFM) with innovative electronic and magnonic band structures. At the same time, they also present new prospects for antiferromagnetic spintronics ${ }^{136}$ with atomically thin engineering of exchange coupling. All these unique aspects of 2D magnets offer opportunities for building novel eFM|NM heterostructure, an open avenue for further exploration and realizing innovative spin devices. Therefore, the very atomically thin nature of the 2D magnets provides multiple prospects that were inconceivable with conventional magnets.

\section{Outlook}

Over the past decade, 2D materials have brought fresh stimuli to the area of magnetic tunnel junctions and different spin injectors. For the first time, we have access to the thinnest materials with minimal interface states and defects, close to perfect systems sought for interfaces. The wide range of electronic structures presented by graphene, hexagonal boron nitride, semiconductors, and their Van der Waals heterostructures, has led to the prediction and demonstration of spin filtering and large magnetoresistance, as elaborated here. The 2D semiconductors also show promise for novel-integration of magnetic junction structures to 2D transistors, signifying a means for integrating nanoelectronic and spintronic devices. One of the striking features of these systems is that even with single 2D layers of spacers, the effects are robust, and noticeably open up opportunities for scientific studies. There exists a vast scope to explore factors such as coupling, hybridization, multiple layers, and hybrid junctions for controlled interfaces to optimize high polarization conditions. As the theoretical results indicate great promise, there is an enormous opportunity for improvements through fabrication and new synthesis techniques for improved figures of merit. At the same time, future theoretical investigations can mine the best combinations that can guide further experiments. Interface spin texture is key to promoting specific phonon spectrum, doping level, and engineering distinct textures through atomic layer twists ${ }^{137}$ and proximity induced spin-orbit coupling ${ }^{138-141}$. For instance, the recent magic-angle bilayer graphene with induced unconventional superconductivity ${ }^{142}$, suggests new feasibility of 2D superconductor-ferromagnet interfaces for superconducting spintronics ${ }^{143}$. Furthermore, the perpendicular magnetic anisotropy (PMA) ${ }^{144,145}$ and Rashba spin-orbit coupling in interfaces with graphene could introduce Dzyaloshinskii-Moriya interaction in adjacent ferromagnetic layers leading to stabilized chiral spin textures ${ }^{146-148}$. Such interfaces could be engineered, and the resulting modification of electronic structure could be quantified experimentally using X-ray as well as transport spectroscopic techniques. Research in this area is expected to evolve rapidly with the recent emergence of ferromagnetism in 2D crystals, as seen from the latest demonstration of 2D Van der Waals based tunnel junctions and the new possibilities with exotic heterostructures. Also, new systems such as mixed dimensional Van der Waals hetrostructures ${ }^{149}$ and $2 D-0 D$ heterostructures ${ }^{150,151}$ provide additional spin-injection and spin-state control knobs via spin-orbit assisted Coulomb effect, enabling the combination of single-electron quantum properties with spin-injection properties. Such inclusion of the single electron mechanism could lead to quantum spin devices with dual electric and magnetic control of spin states. Very recently 2D flexible spin circuits ${ }^{8}$ have also been demonstrated, which bring new aspects for strain-based interface control. Finally, with the possibility of large-scale growth of all such materials through chemical vapor deposition techniques, it is reasonable to expect that a real promise exists for new functional spintronic device applications. 


\section{Acknowledgements}

MVK thanks the support of VR starting Grant (2016-03278) from the Swedish Research Council and funding from the Olle Engkvist foundation, Sweden. JFD thanks financial support of the Institut Universitaire de France, the Agence Nationale de la Recherche under grant Labex NIE 11-LABX0058_NIE within the Investissement d'Avenir program ANR-10-IDEX-0002-02, and under grants MIXES ANR-19-CE09-0028. The authors thanks Pierre Seneor, David Halley, and Bernard Doudin for fruitful discussions.

\section{References}

1. Sanvito, S. Molecular spintronics: The rise of spinterface science. Nat. Phys. 6, 562-564 (2010).

2. Teresa, J. M. De, Barthélémy, A., Fert, A., et al. Role of Metal-Oxide Interface in Determining the Spin Polarization of Magnetic Tunnel Junctions. Science (80-. ). 286, 507-509 (1999).

3. Tsymbal, E. Y., Mryasov, O. N. \& LeClair, P. R. Spin-dependent tunnelling in magnetic tunnel junctions. J. Phys. Condens. Matter 15, R109-R142 (2003).

4. De Teresa, J. M., Barthélémy, A., Fert, A., et al. Inverse Tunnel Magnetoresistance in Co/SrTiO3/La0.7Sr0.3MnO3: New Ideas on Spin-Polarized Tunnelin. Phys. Rev. Lett. 82, 4288-4291 (1999).

5. Roche, S., Åkerman, J., Beschoten, B., et al. Graphene spintronics: The European Flagship perspective. 2D Mater. 2, 030202 (2015).

6. Han, W., Kawakami, R. K., Gmitra, M., et al. Graphene spintronics. Nat. Nanotechnol. 9, 324-340 (2014).

7. Kamalakar, M. V., Groenveld, C., Dankert, A., et al. Long Distance Spin Communication in Chemical Vapour deposited Graphene. Nat. Commun. 6, 6766 (2015).

8. Serrano, I. G., Panda, J., Denoel, F., et al. Two-Dimensional Flexible High Diffusive Spin Circuits. Nano Lett. 19, 666-673 (2019).

9. Avsar, A., Ochoa, H., Guinea, F., et al. Colloquium: Spintronics in graphene and other two-dimensional materials. (2019).

10. Karpan, V. M., Giovannetti, G., Khomyakov, P. ., et al. Graphite and Graphene as Perfect Spin Filters. Phys. Rev. Lett. 99, 176602 (2007).

11. Karpan, V. M., Khomyakov, P. a., Starikov, a. a., et al. Theoretical prediction of perfect spin filtering at interfaces between close-packed surfaces of $\mathrm{Ni}$ or Co and graphite or graphene. Phys. Rev. B 78, 195419 (2008).

12. Yazyev, O. \& Pasquarello, A. Magnetoresistive junctions based on epitaxial graphene and hexagonal boron nitride. Phys. Rev. B 80, 035408 (2009).

13. Dedkov, Y. S., Fonin, M. \& Laubschat, C. A possible source of spin-polarized electrons: The inert graphene/Ni(111) system. Appl. Phys. Lett. 92, 052506 (2008).

14. Dahal, A. \& Batzill, M. Graphene-nickel interfaces: a review. Nanoscale 6, 2548 (2014).

15. Dedkov, Y. S. \& Fonin, M. Electronic and magnetic properties of the graphene-ferromagnet interface. New J. Phys. 12, 125004 (2010).

16. Mohiuddin, T. M. G., Hill, E., Elias, D., et al. Graphene in Multilayered CPP Spin Valves. IEEE Trans. Magn. 44, 2624-2627 (2008).

17. Cobas, E., Friedman, A. L., Van't Erve, O. M. J., et al. Graphene as a tunnel barrier: graphene-based magnetic tunnel junctions. Nano Lett. 12, 3000-4 (2012).

18. Dlubak, B., Martin, M.-B., Weatherup, R. S., et al. Graphene-passivated nickel as an oxidation-resistant electrode for spintronics. ACS Nano 6, 10930-4 (2012).

19. Usachov, D., Fedorov, A., Otrokov, M. M., et al. Observation of Single-Spin Dirac Fermions at the Graphene/Ferromagnet Interface. Nano Lett. 15, 2396-2401 (2015).

20. Zhou, G., Tang, G., Li, T., et al. Graphene-passivated cobalt as a spin-polarized electrode: growth and application to organic spintronics. J. Phys. D. Appl. Phys. 50, 095001 (2017).

21. Li, W., Xue, L., Abruña, H. D., et al. Magnetic tunnel junctions with single-layer-graphene tunnel barriers. Phys. Rev. B 89, 184418 (2014).

22. Park, J.-H. \& Lee, H.-J. Out-of-plane magnetoresistance in ferromagnet/graphene/ferromagnet spinvalve junctions. Phys. Rev. B 89, 165417 (2014).

23. Asshoff, P. U., Sambricio, J. L., Rooney, A. P., et al. Magnetoresistance of vertical Co-graphene-NiFe junctions controlled by charge transfer and proximity-induced spin splitting in graphene. 2D Mater. 4, 
031004 (2017).

24. Piquemal-Banci, M., Galceran, R., Martin, M.-B., et al. 2D-MTJs: introducing 2D materials in magnetic tunnel junctions. J. Phys. D. Appl. Phys. 50, 203002 (2017).

25. Iqbal, M. Z., Qureshi, N. A. \& Hussain, G. Recent advancements in 2D-materials interface based magnetic junctions for spintronics. J. Magn. Magn. Mater. 457, 110-125 (2018).

26. Martin, M.-B., Dlubak, B., Weatherup, R. S., et al. Sub-nanometer Atomic Layer Deposition for Spintronics in Magnetic Tunnel Junctions Based on Graphene Spin-Filtering Membranes. ACS Nano (2014). doi:10.1021/nn5017549

27. Cobas, E. D., van't Erve, O. M. J., Cheng, S.-F., et al. Room-Temperature Spin Filtering in Metallic Ferromagnet-Multilayer Graphene-Ferromagnet Junctions. ACS Nano 10, 10357-10365 (2016).

28. Godel, F., Kamalakar, M. V., Doudin, B., et al. Voltage-controlled inversion of tunnel magnetoresistance in epitaxial nickel/graphene/MgO/cobalt junctions. Appl. Phys. Lett. 105, 152407 (2014).

29. Martin, M.-B., Dlubak, B., Weatherup, R. S., et al. Protecting nickel with graphene spin-filtering membranes: A single layer is enough. Appl. Phys. Lett. 107, 012408 (2015).

30. Singh, A. K. \& Eom, J. Negative magnetoresistance in a vertical single-layer graphene spin valve at room temperature. ACS Appl. Mater. Interfaces 6, 2493-6 (2014).

31. Chen, J.-J., Meng, J., Zhou, Y.-B., et al. Layer-by-layer assembly of vertically conducting graphene devices. Nat. Commun. 4, 1921 (2013).

32. Iqbal, M. Z., Iqbal, M. W., Lee, J. H., et al. Spin valve effect of NiFe/graphene/NiFe junctions. Nano Res. 6, 373-380 (2013).

33. Meng, J., Chen, J.-J., Yan, Y., et al. Vertical graphene spin valve with Ohmic contacts. Nanoscale 5, 8894 (2013).

34. Iqbal, M. Z., Iqbal, M. W., Jin, X., et al. Interlayer dependent polarity of magnetoresistance in graphene spin valves. J. Mater. Chem. C 3, 298-302 (2015).

35. Iqbal, M. Z., Hussain, G., Siddique, S., et al. Interlayer reliant magnetotransport in graphene spin valve. J. Magn. Magn. Mater. 441, 39-42 (2017).

36. Entani, S., Seki, T., Sakuraba, Y., et al. Magnetoresistance effect in $\mathrm{Fe}_{20} \mathrm{Ni} 80$ /graphene/Fe $20 \mathrm{Ni}_{80}$ vertical spin valves. Appl. Phys. Lett. 109, 082406 (2016).

37. Julliere, M. Tunneling between ferromagnetic films. Phys. Lett. A 54, 225-226 (1975).

38. Zhang, Y., Brar, V. W., Wang, F., et al. Giant phonon-induced conductance in scanning tunnelling spectroscopy of gate-tunable graphene. Nat. Phys. 4, 627-630 (2008).

39. Halle, J., Néel, N., Fonin, M., et al. Understanding and Engineering Phonon-Mediated Tunneling into Graphene on Metal Surfaces. Nano Lett. 18, 5697-5701 (2018).

40. Allard, A. \& Wirtz, L. Graphene on Metallic Substrates: Suppression of the Kohn Anomalies in the Phonon Dispersion. Nano Lett. 10, 4335-4340 (2010).

41. Voloshina, E. N., Generalov, A., Weser, M., et al. Structural and electronic properties of the graphene/Al/Ni(111) intercalation system. New J. Phys. 13, 113028 (2011).

42. Néel, N., Steinke, C., Wehling, T. O., et al. Inelastic electron tunneling into graphene nanostructures on a metal surface. Phys. Rev. B 95, 161410 (2017).

43. Žutić, I., Matos-Abiague, A., Scharf, B., et al. Proximitized materials. Mater. Today 22, 85-107 (2019).

44. Gmitra, M., Kochan, D., Högl, P., et al. Trivial and inverted Dirac bands and the emergence of quantum spin Hall states in graphene on transition-metal dichalcogenides. Phys. Rev. B 93, 155104 (2016).

45. Avsar, A., Tan, J. Y., Taychatanapat, T., et al. Spin-orbit proximity effect in graphene. Nat. Commun. 5, 4875 (2014).

46. Safeer, C. K., Ingla-Aynés, J., Herling, F., et al. Room-Temperature Spin Hall Effect in Graphene/MoS 2 van der Waals Heterostructures. Nano Lett. 19, 1074-1082 (2019).

47. Gmitra, M. \& Fabian, J. Graphene on transition-metal dichalcogenides: A platform for proximity spinorbit physics and optospintronics. Phys. Rev. B - Condens. Matter Mater. Phys. 92, 1-6 (2015).

48. Luo, Y. K., Xu, J., Zhu, T., et al. Opto-Valleytronic Spin Injection in Monolayer MoS 2 /Few-Layer Graphene Hybrid Spin Valves. Nano Lett. 17, 3877-3883 (2017).

49. Avsar, A., Unuchek, D., Liu, J., et al. Optospintronics in Graphene via Proximity Coupling. ACS Nano 11, 11678-11686 (2017).

50. Qiao, Z., Ren, W., Chen, H., et al. Quantum Anomalous Hall Effect in Graphene Proximity Coupled to an Antiferromagnetic Insulator. Phys. Rev. Lett. 112, 116404 (2014).

51. Wei, P., Lee, S., Lemaitre, F., et al. Strong interfacial exchange field in the graphene/EuS heterostructure. Nat. Mater. 15, 711-716 (2016).

52. Wang, Z., Tang, C., Sachs, R., et al. Proximity-induced ferromagnetism in graphene revealed by the 
anomalous hall effect. Phys. Rev. Lett. 114, 1-16 (2015).

53. Zollner, K., Gmitra, M., Frank, T., et al. Theory of proximity-induced exchange coupling in graphene on hBN/(Co, Ni). Phys. Rev. B 94, 155441 (2016).

54. Zollner, K., Faria Junior, P. E. \& Fabian, J. Proximity exchange effects in MoSe 2 and WSe 2 heterostructures with $\mathrm{Crl} 3$ : Twist angle, layer, and gate dependence. Phys. Rev. B 100, 085128 (2019).

55. Cardoso, C., Soriano, D., García-Martínez, N. A., et al. Van der Waals Spin Valves. Phys. Rev. Lett. 121, 067701 (2018).

56. Yuasa, S. \& Djayaprawira, D. D. Giant tunnel magnetoresistance in magnetic tunnel junctions with a crystalline MgO(0 0 1) barrier. J. Phys. D. Appl. Phys. 40, (2007).

57. Gao, K. Z., Heinonen, O. \& Chen, Y. Read and write processes, and head technology for perpendicular recording. J. Magn. Magn. Mater. 321, 495-507 (2009).

58. Britnell, L., Gorbachev, R. V, Jalil, R., et al. Electron tunneling through ultrathin boron nitride crystalline barriers. Nano Lett. 12, 1707-1710 (2012).

59. Yamaguchi, T., Inoue, Y., Masubuchi, S., et al. Electrical Spin Injection into Graphene through Monolayer Hexagonal Boron Nitride. Appl. Phys. Express 6, 073001 (2013).

60. Kamalakar, M. V., Dankert, A., Bergsten, J., et al. Enhanced Tunnel Spin Injection into Graphene using Chemical Vapor Deposited Hexagonal Boron Nitride. Sci. Rep. 4, 6146 (2014).

61. Fu, W., Makk, P., Bräuninger, M., et al. Large-scale fabrication of BN tunnel barriers for graphene spintronics. J. Appl. Phys. 116, 074306 (2014).

62. Kamalakar, M. V., Dankert, A., Bergsten, J., et al. Spintronics with graphene-hexagonal boron nitride van der Waals heterostructures. Appl. Phys. Lett. 105, 212405 (2014).

63. Karpan, V. M., Khomyakov, P. A., Giovannetti, G., et al. Ni(111) | graphene|h-BN junctions as ideal spin injectors. Phys. Rev. B 84, 153406 (2011).

64. Wu, Q., Shen, L., Bai, Z., et al. Efficient Spin Injection into Graphene through a Tunnel Barrier: Overcoming the Spin-Conductance Mismatch. Phys. Rev. Appl. 2, 044008 (2014).

65. Kamalakar, M. V., Dankert, A., Kelly, P. J., et al. Inversion of Spin Signal and Spin Filtering in Ferromagnet|Hexagonal Boron Nitride-Graphene van der Waals Heterostructures. Sci. Rep. 6, 21168 (2016).

66. Iqbal, M. Z., Siddique, S. \& Hussain, G. Spin Valve Effect of 2D-Materials Based Magnetic Junctions. Adv. Eng. Mater. 20, 1-6 (2018).

67. Piquemal-Banci, M., Galceran, R., Godel, F., et al. Insulator-to-Metallic Spin-Filtering in 2D-Magnetic Tunnel Junctions Based on Hexagonal Boron Nitride. ACS Nano 12, 4712-4718 (2018).

68. Asshoff, P. U., Sambricio, J. L., Slizovskiy, S., et al. Magnetoresistance in Co-hBN-NiFe Tunnel Junctions Enhanced by Resonant Tunneling through Single Defects in Ultrathin hBN Barriers. Nano Lett. 18, 69546960 (2018).

69. Dankert, A., Kamalakar, M. V., Wajid, A., et al. Tunnel magnetoresistance with atomically thin two dimensional hexagonal boron nitride barriers. Nano Res. 8, 1357-1364 (2014).

70. Piquemal-Banci, M., Galceran, R., Caneva, S., et al. Magnetic tunnel junctions with monolayer hexagonal boron nitride tunnel barriers. Appl. Phys. Lett. 108, 1-4 (2016).

71. Tarawneh, K., Al-Aqtash, N. \& Sabirianov, R. Large magnetoresistance in planar Fe/MoS2/Fe tunnel junction. Comput. Mater. Sci. 124, 15-22 (2016).

72. Kumar, A. Enhanced Magnetoresistance in In-Plane Monolayer MoS 2 with CrO 2 Electrodes. 2-7 (2018).

73. Wong, W. C., Ng, S. M., Wong, H. F., et al. Spin-Valve Junction With Transfer-Free. 53, (2017).

74. Dankert, A., Pashaei, P., Kamalakar, M. V., et al. Spin-Polarized Tunneling through Chemical Vapor Deposited Multilayer Molybdenum Disulfide. ACS Nano 11, 6389-6395 (2017).

75. Zhang, H., Ye, M., Wang, Y., et al. Magnetoresistance in Co/2D MoS2/Co and Ni/2D MoS2/Ni junctions. Phys. Chem. Chem. Phys. 18, 16367-16376 (2016).

76. Dolui, K., Narayan, A., Rungger, I., et al. Efficient spin injection and giant magnetoresistance in Fe/MoS2/Fe junctions. Phys. Rev. B - Condens. Matter Mater. Phys. 90, 1-5 (2014).

77. Rotjanapittayakul, W., Pijitrojana, W., Archer, T., et al. Spin injection and magnetoresistance in MoS2based tunnel junctions using Fe3Si Heusler alloy electrodes. Sci. Rep. 8, 1-8 (2018).

78. Wu, H.-C., Coileáin, C. Ó., Abid, M., et al. Spin-dependent transport properties of Fe304/MoS2/Fe304 junctions. Sci. Rep. 5, 15984 (2015).

79. Khan, M. F., Kim, H., Nazir, G., et al. Layer dependent magnetoresistance of vertical MoS $_{2}$ magnetic tunnel junctions. Nanoscale (2018). doi:10.1039/C8NR04518F 
80. Zhang, Y., Ning, H., Li, Y., et al. Negative to positive crossover of the magnetoresistance in layered WS2. Appl. Phys. Lett. 108, 1-5 (2016).

81. Iqbal, M. Z., Iqbal, M. W., Siddique, S., et al. Room temperature spin valve effect in NiFe/WS2/Co junctions. Sci. Rep. 6, 21038 (2016).

82. Zhao, K., Xing, Y., Han, J., et al. Magnetic transport property of NiFe/WSe2/NiFe spin valve structure. J. Magn. Magn. Mater. 432, 10-13 (2017).

83. Zhang, E., Chen, R., Huang, C., et al. Tunable Positive to Negative Magnetoresistance in Atomically Thin WTe2. Nano Lett. 17, 878-885 (2017).

84. Wang, W., Narayan, A., Tang, L., et al. Spin-Valve Effect in NiFe/MoS 2 /NiFe Junctions. Nano Lett. 150710123919002 (2015). doi:10.1021/acs.nanolett.5b01553

85. Mouafo, L. D. N., Godel, F., Froehlicher, G., et al. Tuning contact transport mechanisms in bilayer MoSe ${ }_{2}$ transistors up to Fowler-Nordheim regime. 2D Mater. 4, 015037 (2016).

86. Moodera, J. S., Kinder, L. R., Wong, T. M., et al. Large Magnetoresistance at Room Temperature in Ferromagnetic Thin Film Tunnel Junctions. Phys. Rev. Lett. 74, 3273-3276 (1995).

87. Miyazaki, T. \& Tezuka, N. Giant magnetic tunneling effect in Fe/Al2O3/Fe junction. J. Magn. Magn. Mater. 139, L231-L234 (1995).

88. Chappert, C., Fert, A. \& Van Dau, F. N. The emergence of spin electronics in data storage. Nat. Mater. 6, 813-823 (2007).

89. Falicov, L. M., Pierce, D. T., Bader, S. D., et al. Surface, interface, and thin-film magnetism. J. Mater. Res. 5, 1299-1340 (1990).

90. Zhu, J.-G. (Jimmy) \& Park, C. Magnetic tunnel junctions. Mater. Today 9, 36-45 (2006).

91. Martin, L. W., Chu, Y.-H. \& Ramesh, R. Advances in the growth and characterization of magnetic, ferroelectric, and multiferroic oxide thin films. Mater. Sci. Eng. R Reports 68, 89-133 (2010).

92. Cardoso, S., Freitas, P. P., de Jesus, C., et al. Spin-tunnel-junction thermal stability and interface interdiffusion above $300^{\circ} \mathrm{C}$. Appl. Phys. Lett. 76, 610-612 (2000).

93. Shen, W., Mazumdar, D., Zou, X., et al. Effect of film roughness in MgO-based magnetic tunnel junctions. Appl. Phys. Lett. 88, 182508 (2006).

94. Da Costa, V., Tiusan, C., Dimopoulos, T., et al. Tunneling Phenomena as a Probe to Investigate Atomic Scale Fluctuations in Metal/Oxide/Metal Magnetic Tunnel Junctions. Phys. Rev. Lett. 85, 876-879 (2000).

95. Costa, V. Da, Henry, Y., Bardou, F., et al. Experimental evidence and consequences of rare events in quantum tunneling. Eur. Phys. J. B 13, 297-303 (2000).

96. Tsymbal, E. Y. \& Pettifor, D. G. Spin-polarized electron tunneling across a disordered insulator. Phys. Rev. B 58, 432-437 (1998).

97. Velev, J. P., Belashchenko, K. D., Jaswal, S. S., et al. Effect of oxygen vacancies on spin-dependent tunneling in Fe/MgO/Fe magnetic tunnel junctions. Appl. Phys. Lett. 90, 072502 (2007).

98. Beaufrand, J.-B., Dayen, J.-F., Kemp, N. T., et al. Magnetoresistance signature of resonant states in electromigrated Ni nanocontacts. Appl. Phys. Lett. 98, 142504 (2011).

99. Tsymbal, E., Sokolov, a., Sabirianov, I., et al. Resonant Inversion of Tunneling Magnetoresistance. Phys. Rev. Lett. 90, 186602 (2003).

100. Levy, P. M., Wang, K., Dederichs, P. H., et al. An approximate calculation for transport in magnetic tunnel junctions in the presence of localized states. Philos. Mag. B 82, 763-769 (2002).

101. Uiberacker, C. \& Levy, P. M. Role of symmetry on interface states in magnetic tunnel junctions. Phys. Rev. B 64, 193404 (2001).

102. Martins, L., Ventura, J., Ferreira, R., et al. Optimization of the buffer surface of CoFeB/MgO/CoFeBbased magnetic tunnel junctions by ion beam milling. Appl. Surf. Sci. 424, 58-62 (2017).

103. Tsymbal, E., Belashchenko, K., Velev, J., et al. Interface effects in spin-dependent tunneling. Prog. Mater. Sci. 52, 401-420 (2007).

104. Geim, A. K. \& Grigorieva, I. V. Van der Waals heterostructures. Nature 499, 419-25 (2013).

105. Liu, Y., Weiss, N. O., Duan, X., et al. Van der Waals heterostructures and devices. Nat. Rev. Mater. 1, natrevmats201642 (2016).

106. Li, X., Lü, J.-T., Zhang, J., et al. Spin-Dependent Transport in van der Waals Magnetic Tunnel Junctions with $\mathrm{Fe}_{3} \mathrm{GeTe}_{2}$ Electrodes. Nano Lett. 19, 5133-5139 (2019).

107. Wang, Z., Sapkota, D., Taniguchi, T., et al. Tunneling Spin Valves Based on $\mathrm{Fe}_{3} \mathrm{GeTe}_{2} / \mathrm{hBN} / \mathrm{Fe}_{3} \mathrm{GeTe}_{2}$ van der Waals Heterostructures. Nano Lett. 18, 4303-4308 (2018).

108. Klein, D. R., MacNeill, D., Lado, J. L., et al. Probing magnetism in 2D van der Waals crystalline insulators via electron tunneling. Science (80-. ). 3617, 1-9 (2018). 
109. Paudel, T. R. \& Tsymbal, E. Y. Spin Filtering in $\mathrm{Crl}_{3}$ Tunnel Junctions. ACS Appl. Mater. Interfaces 11, 15781-15787 (2019).

110. Song, T., Cai, X., Tu, M. W.-Y., et al. Giant tunneling magnetoresistance in spin-filter van der Waals heterostructures. Science 360, 1214-1218 (2018).

111. Mermin, N. D. \& Wagner, H. Absence of Ferromagnetism or Antiferromagnetism in One- or TwoDimensional Isotropic Heisenberg Models. Phys. Rev. Lett. 17, 1133-1136 (1966).

112. Carteaux, V., Moussa, F. \& Spiesser, M. 2d ising-like ferromagnetic behaviour for the lamellar cr2si2te6 compound: A neutron scattering investigation. Ep/ 29, 251-256 (1995).

113. Gong, C., Li, L., Li, Z., et al. Discovery of intrinsic ferromagnetism in two-dimensional van der Waals crystals. Nature 546, 265-269 (2017).

114. Huang, B., Clark, G., Navarro-Moratalla, E., et al. Layer-dependent ferromagnetism in a van der Waals crystal down to the monolayer limit. Nature 546, 270-273 (2017).

115. Bonilla, M., Kolekar, S., Ma, Y., et al. Strong room-temperature ferromagnetism in VSe2 monolayers on van der Waals substrates. Nat. Nanotechnol. (2018). doi:10.1038/s41565-018-0063-9

116. Lebègue, S., Björkman, T., Klintenberg, M., et al. Two-dimensional materials from data filtering and $A b$ Initio calculations. Phys. Rev. X 3, 1-7 (2013).

117. Fei, Z., Huang, B., Malinowski, P., et al. Two-Dimensional Itinerant Ising Ferromagnetism in Atomically thin Fe3GeTe2. Nat. Mater. 17, (2018).

118. Xing, W., Chen, Y., Odenthal, P. M., et al. Electric field effect in multilayer Cr 2 Ge 2 Te 6: a ferromagnetic 2D material. 2D Mater. 4, 024009 (2017).

119. Huang, B., Clark, G., Klein, D. R., et al. Electrical control of 2D magnetism in bilayer CrI3. Nat. Nanotechnol. 13, 544-548 (2018).

120. Wang, Z., Gutiérrez-Lezama, I., Ubrig, N., et al. Very large tunneling magnetoresistance in layered magnetic semiconductor Crl3. Nat. Commun. 9, (2018).

121. Ghazaryan, D., Greenaway, M. T., Wang, Z., et al. Magnon-assisted tunnelling in van der Waals heterostructures based on $\mathrm{CrBr}$ 3. Nat. Electron. 1, 344-349 (2018).

122. Tan, C., Lee, J., Jung, S.-G., et al. Hard magnetic properties in nanoflake van der Waals Fe3GeTe2. Nat. Commun. 9, 1554 (2018).

123. Lin, M. W., Zhuang, H. L., Yan, J., et al. Ultrathin nanosheets of CrSiTe3: A semiconducting twodimensional ferromagnetic material. J. Mater. Chem. C 4, 315-322 (2016).

124. Jiang, S., Li, L., Wang, Z., et al. Controlling magnetism in 2D Crl3 by electrostatic doping. Nat. Nanotechnol. 13, 549-553 (2018).

125. Jiang, S., Shan, J. \& Mak, K. F. Electric-field switching of two-dimensional van der Waals magnets. Nat. Mater. 17, 406-410 (2018).

126. Zhong, D., Seyler, K. L., Linpeng, X., et al. Van der Waals engineering of ferromagnetic semiconductor heterostructures for spin and valleytronics. Sci. Adv. 3, e1603113 (2017).

127. Alegria, L. D., Ji, H., Yao, N., et al. Large anomalous Hall effect in ferromagnetic insulator-topological insulator heterostructures. Appl. Phys. Lett. 105, 053512 (2014).

128. Katmis, F., Lauter, V., Nogueira, F. S., et al. A high temperature ferromagnetic topological insulating phase by proximity coupling. Nature 533, 513-516 (2016).

129. Sivadas, N., Okamoto, S., Xu, X., et al. Stacking-Dependent Magnetism in Bilayer $\mathrm{Crl}_{3}$. Nano Lett. 18, 7658-7664 (2018).

130. Jiang, P., Wang, C., Chen, D., et al. Stacking tunable interlayer magnetism in bilayer Crl 3. Phys. Rev. B 99, 144401 (2019).

131. Soriano, D., Cardoso, C. \& Fernández-Rossier, J. Interplay between interlayer exchange and stacking in Crl3 bilayers. Solid State Commun. 299, 113662 (2019).

132. Weber, D., Schoop, L. M., Duppel, V., et al. Magnetic Properties of Restacked 2D Spin 1/2 honeycomb $\mathrm{RuCl}_{3}$ Nanosheets. Nano Lett. 16, 3578-3584 (2016).

133. Klein, D. R., MacNeill, D., Song, Q., et al. Enhancement of interlayer exchange in an ultrathin twodimensional magnet. Nat. Phys. 1-6 (2019). doi:10.1038/s41567-019-0651-0

134. Song, T., Fei, Z., Yankowitz, M., et al. Switching 2D Magnetic States via Pressure Tuning of Layer Stacking. (2019).

135. Chen, W., Sun, Z., Gu, L., et al. Direct observation of van der Waals stacking dependent interlayer magnetism. (2019).

136. Jungwirth, T., Sinova, J., Manchon, A., et al. The multiple directions of antiferromagnetic spintronics. Nat. Phys. 14, 200-203 (2018).

137. Song, J. C. W. \& Gabor, N. M. Electron quantum metamaterials in van der Waals heterostructures. Nat. 
Nanotechnol. 13, (2018).

138. Henck, H., Ben Aziza, Z., Pierucci, D., et al. Electronic band structure of Two-Dimensional WS 2 /Graphene van der Waals Heterostructures. Phys. Rev. B 97, 155421 (2018).

139. Wakamura, T., Reale, F., Palczynski, P., et al. Spin-Orbit Interaction Induced in Graphene by TransitionMetal Dichalcogenides. (2018).

140. Cummings, A. W., Garcia, J. H., Fabian, J., et al. Giant Spin Lifetime Anisotropy in Graphene Induced by Proximity Effects. Phys. Rev. Lett. 119, 206601 (2017).

141. Song, K., Soriano, D., Cummings, A. W., et al. Spin Proximity Effects in Graphene/Topological Insulator Heterostructures. Nano Lett. 18, 2033-2039 (2018).

142. Cao, Y., Fatemi, V., Fang, S., et al. Unconventional superconductivity in magic-angle graphene superlattices. Nature 556, 43-50 (2018).

143. Linder, J. \& Robinson, J. W. A. Superconducting spintronics. Nat. Phys. 11, 307-315 (2015).

144. Vo-Van, C., Kassir-Bodon, Z., Yang, H., et al. Ultrathin epitaxial cobalt films on graphene for spintronic investigations and applications. New J. Phys. 12, 103040 (2010).

145. Rougemaille, N., N’Diaye, A. T., Coraux, J., et al. Perpendicular magnetic anisotropy of cobalt films intercalated under graphene. Appl. Phys. Lett. 101, 142403 (2012).

146. Yang, H., Chen, G., Cotta, A. A. C., et al. Significant Dzyaloshinskii-Moriya interaction at grapheneferromagnet interfaces due to the Rashba effect. Nat. Mater. 17, 605-609 (2018).

147. Chaurasiya, A. K., Kumar, A., Gupta, R., et al. Direct observation of unusual interfacial DzyaloshinskiiMoriya interaction in graphene/NiFe/Ta heterostructures. Phys. Rev. B 99, 1-6 (2019).

148. Ajejas, F., Gudín, A., Guerrero, R., et al. Unraveling Dzyaloshinskii-Moriya Interaction and Chiral Nature of Graphene/Cobalt Interface. Nano Lett. 18, 5364-5372 (2018).

149. Jariwala, D., Marks, T. J. \& Hersam, M. C. Mixed-dimensional van der Waals heterostructures. Nat. Mater. 16, 170-181 (2017).

150. Mouafo, L. D. N., Godel, F., Melinte, G., et al. Anisotropic Magneto-Coulomb Properties of 2D-0D Heterostructure Single Electron Device. Adv. Mater. 1802478 (2018). doi:10.1002/adma.201802478

151. Godel, F., Mouafo, L. D. N., Froehlicher, G., et al. Conductance Oscillations in a Graphene/Nanocluster Hybrid Material: Toward Large-Area Single-Electron Devices. Adv. Mater. 29, 1604837 (2017). 